\title{
A restricted Epstein zeta function and the evaluation of some definite integrals
}

\author{
by \\ Habib Muzaffar and Kenneth S. Williams (Ottawa)
}

1. Introduction. A nonzero integer $d$ is called a discriminant if $d \equiv 0$ or $1(\bmod 4)$. We set

$$
d=\Delta(d) f(d)^{2},
$$

where $f(d)$ is the largest positive integer for which $\Delta(d)=d / f(d)^{2}$ is a discriminant. The integer $f(d)$ is called the conductor of the discriminant $d$. The discriminant $d$ is called fundamental if $f(d)=1$. A discriminant $d$ is fundamental if and only if $d$ is odd and squarefree or $d$ is even, $d / 4$ is squarefree and $d / 4 \equiv 2$ or $3(\bmod 4)$. We note that the discriminant $\Delta(d)$ is fundamental so that $f(\Delta(d))=1$ and $\Delta(\Delta(d))=\Delta(d)$. If $d=\Delta^{\prime} f^{\prime 2}$, where $\Delta^{\prime}$ is a fundamental discriminant and $f^{\prime}$ is a positive integer, then $\Delta^{\prime}=\Delta(d)$ and $f^{\prime}=f(d)$. The discriminant $\Delta(d)$ is called the fundamental discriminant associated with the discriminant $d$. If $d_{1}$ and $d_{2}$ are discriminants then $d_{1} d_{2}$ is also a discriminant. We have

$d_{1}=\Delta\left(d_{1}\right) f\left(d_{1}\right)^{2}, \quad d_{2}=\Delta\left(d_{2}\right) f\left(d_{2}\right)^{2}, \quad d_{1} d_{2}=\Delta\left(d_{1}\right) \Delta\left(d_{2}\right)\left(f\left(d_{1}\right) f\left(d_{2}\right)\right)^{2}$

so that

$$
d_{1} d_{2}=\Delta\left(\Delta\left(d_{1}\right) \Delta\left(d_{2}\right)\right)\left(f\left(\Delta\left(d_{1}\right) \Delta\left(d_{2}\right)\right) f\left(d_{1}\right) f\left(d_{2}\right)\right)^{2},
$$

and thus

$$
\Delta\left(d_{1} d_{2}\right)=\Delta\left(\Delta\left(d_{1}\right) \Delta\left(d_{2}\right)\right), \quad f\left(d_{1} d_{2}\right)=f\left(\Delta\left(d_{1}\right) \Delta\left(d_{2}\right)\right) f\left(d_{1}\right) f\left(d_{2}\right) .
$$

In particular we have

$$
f\left(d_{1}\right)\left|f\left(d_{1} d_{2}\right), \quad f\left(d_{2}\right)\right| f\left(d_{1} d_{2}\right) .
$$

2000 Mathematics Subject Classification: Primary 11E16, 11E25, 11E45; Secondary $33 \mathrm{~B} 10$.

Key words and phrases: binary quadratic forms, genera, Chowla-Selberg formula, definite integrals.

Research of the second author was supported by Natural Sciences and Engineering Research Council of Canada grant A-7233. 
If $k$ is a positive integer then $k^{2}$ is a discriminant with $\Delta\left(k^{2}\right)=1, f\left(k^{2}\right)=k$, so that for any discriminant $d$ we have

$$
\begin{aligned}
\Delta\left(d k^{2}\right) & =\Delta\left(\Delta(d) \Delta\left(k^{2}\right)\right)=\Delta(\Delta(d))=\Delta(d), \\
f\left(d k^{2}\right) & =f\left(\Delta(d) \Delta\left(k^{2}\right)\right) f(d) f\left(k^{2}\right)=f(\Delta(d)) f(d) k=f(d) k .
\end{aligned}
$$

When there is no confusion, we write $\Delta=\Delta(d)$ and $f=f(d)$.

Throughout the rest of this paper, $d$ represents a nonsquare discriminant and $n$ represents a positive integer. For integers $a, b$ and $c$ with $\operatorname{gcd}(a, b, c)$ $=1$, we use $(a, b, c)$ to denote the primitive, integral, binary quadratic form $a x^{2}+b x y+c y^{2}$. A form $(a, b, c)$ with $b^{2}-4 a c=d$ is called a form of discriminant $d$. Such a form is irreducible in $\mathbb{Z}[x, y]$ as $d$ is not a square. Two forms $(a, b, c)$ and $\left(a^{\prime}, b^{\prime}, c^{\prime}\right)$ are equivalent if and only if there exist integers $r, s, t$ and $u$ with $r u-s t=1$ such that the substitution $x=r X+s Y, y=$ $t X+u Y$ transforms $(a, b, c)$ to $\left(a^{\prime}, b^{\prime}, c^{\prime}\right)$. If $(a, b, c)$ is equivalent to $\left(a^{\prime}, b^{\prime}, c^{\prime}\right)$, we write $(a, b, c) \sim\left(a^{\prime}, b^{\prime}, c^{\prime}\right)$. The relation $\sim$ is an equivalence relation on the set of forms of discriminant $d$. We denote the class of $(a, b, c)$ by $[a, b, c]$. The classes of primitive, integral, binary quadratic forms of discriminant $d$ (only positive-definite forms are used if $d<0$ ) form a finite abelian group under Gaussian composition (see for example [1: Chapter 4]). We denote this group by $H(d)$ and its order by $h(d)$. The cosets of the subgroup of squares in $H(d)$ are called genera and we denote the group of genera by $G(d)$. The identity element of $G(d)$ is called the principal genus. By group theory we have $|G(d)|=2^{t}$, where $t=t(d)$ is a nonnegative integer. The value of $t(d)$ is given by [7: $\S 153$, pp. 409-413; $\S 151$, pp. 400-407] (see also [13: p. 277])

$$
t(d)= \begin{cases}\omega(d) & \text { if } d \equiv 0(\bmod 32) \\ \omega(d)-2 & \text { if } d \equiv 4(\bmod 16) \\ \omega(d)-1 & \text { otherwise }\end{cases}
$$

where $\omega(d)$ denotes the number of distinct prime factors of $d$. Thus $|G|=$ $h(d) / 2^{t}$ for any $G \in G(d)$.

Let $[a, b, c] \in H(d)$. The positive integer $n$ is said to be represented by the form $(a, b, c)$ if there exist integers $x$ and $y$ with $a x^{2}+b x y+c y^{2}=n$, and the pair $(x, y)$ is called a representation. If $d<0$ every representation $(x, y)$ is called primary. If $d>0$ the representation $(x, y)$ is called primary if it satisfies

$$
2 a x+(b-\sqrt{d}) y>0 \quad \text { and } \quad 1 \leq\left|\frac{2 a x+(b+\sqrt{d}) y}{2 a x+(b-\sqrt{d}) y}\right|<\varepsilon^{2},
$$

where

$$
\varepsilon=\varepsilon(d)=\left(x_{0}+y_{0} \sqrt{d}\right) / 2,
$$

and $\left(x_{0}, y_{0}\right)=\left(x_{0}(d), y_{0}(d)\right)$ is the solution in positive integers to the equa- 
tion $x^{2}-d y^{2}=4$ for which $y_{0}$ is least (see for example [12: p. 282]). We set

(4) $\quad R_{(a, b, c)}(n, d)=\operatorname{card}\left\{(x, y) \in \mathbb{Z}^{2}: a x^{2}+b x y+c y^{2}=n,(x, y)\right.$ primary $\}$. $R_{(a, b, c)}(n, d)$ is finite and $R_{(a, b, c)}(n, d)=R_{\left(a^{\prime}, b^{\prime}, c^{\prime}\right)}(n, d)$ if $(a, b, c) \sim\left(a^{\prime}, b^{\prime}, c^{\prime}\right)$ (see for example [12: $\S 11.4]$ ). Thus we can define

$$
R_{[a, b, c]}(n, d)=R_{(a, b, c)}(n, d) .
$$

For $G \in G(d)$, we set

$$
R_{G}(n, d)=\sum_{K \in G} R_{K}(n, d) .
$$

When $d<0$, Huard, Kaplan and Williams [13: Theorem 8.1] have obtained an explicit formula for $R_{G}(n, d)$. Using this formula they showed [13: Theorem 10.2] that for $s \rightarrow 1^{+}$,

$$
\sum_{n=1}^{\infty} \frac{R_{G}(n, d)}{n^{s}}=\frac{h(d)}{2^{t(d)}} \cdot \frac{2 \pi}{\sqrt{|d|}} \cdot \frac{1}{s-1}+B_{G}(d)+O(s-1),
$$

where $B_{G}(d)$ is an explicit constant depending on $d$ and $G$. In this paper, we extend their ideas to the case $d>0$. In Section 2 we obtain a formula for $R_{G}(n, d)$ when $d>0$ (see Theorem 1). In Section 4 we use this formula to determine $\sum_{n=1}^{\infty} R_{G}(n, d) / n^{s}$ for $d>0$ and $s>1$ (see Theorem 3). From Theorem 3 we deduce that

$$
\sum_{n=1}^{\infty} \frac{R_{G}(n, d)}{n^{s}}=\frac{h(d)}{2^{t(d)}} \cdot \frac{\log \varepsilon(d)}{\sqrt{d}} \cdot \frac{1}{s-1}+B(d)+\beta(d, G)+O(s-1),
$$

where $B(d)$ is a constant depending only on $d$ and not on $G$ and $\beta(d, G)$ is an explicit constant depending on both $d$ and $G$ (see Theorem 4).

If $Q=(a, b, c)$ is a positive-definite binary quadratic form of discriminant $d<0$, the Epstein zeta function $Z_{Q}(s)$ corresponding to $Q$ is defined for $s>1$ by the infinite series

$$
Z_{Q}(s)=\sum_{\substack{x, y=-\infty \\(x, y) \neq(0,0)}}^{\infty} \frac{1}{Q(x, y)^{s}}
$$

(see for example [5], [8], [14], [15]). The behaviour of $Z_{Q}(s)$ near $s=1$ is given by Kronecker's limit formula (see for example [13: p. 300], [15: p. 14])

$$
Z_{Q}(s)=\frac{2 \pi}{\sqrt{|d|}} \cdot \frac{1}{s-1}+K(a, b, c)+O(s-1),
$$

where $K(a, b, c)$ is an explicit constant depending only on $a, b$ and $c$. Let 
$G \in G(d)$. As

$$
\sum_{n=1}^{\infty} \frac{R_{G}(n, d)}{n^{s}}=\sum_{[a, b, c] \in G} Z_{(a, b, c)}(s)
$$

we obtain

$$
\begin{aligned}
\frac{h(d)}{2^{t(d)}} \cdot \frac{2 \pi}{\sqrt{|d|}} \cdot \frac{1}{s-1} & +B_{G}(d)+O(s-1) \\
& =\sum_{[a, b, c] \in G}\left(\frac{2 \pi}{\sqrt{|d|}} \cdot \frac{1}{s-1}+K(a, b, c)+O(s-1)\right),
\end{aligned}
$$

so that

$$
B_{G}(d)=\sum_{[a, b, c] \in G} K(a, b, c) .
$$

The Chowla-Selberg formula for genera, which was proved by Huard, Kaplan and Williams [13: Theorem 1.1] in 1995, is obtained by putting the explicit values of $B_{G}(d)$ and $K(a, b, c)$ into (11) and exponentiating the resulting formula.

We now define an analogue of the Epstein zeta function (9) in the case of an indefinite binary quadratic form $Q=(a, b, c)$ of discriminant $d>0$ with $a>0$. We remark that if the form $(a, b, c)$ is indefinite, then we can always replace it by an equivalent one with $a>0$. To see this, recall that an indefinite form $(a, b, c)$ represents both positive and negative integers. Let $k$ be a positive integer represented by $(a, b, c)$. Then there is a positive integer $l$ dividing $k$ which is properly represented by $(a, b, c)$. Hence $(a, b, c) \sim\left(l, b^{\prime}, c^{\prime}\right)$ for some integers $b^{\prime}$ and $c^{\prime}$. We call our analogue of (9) the restricted Epstein zeta function and denote it by $Z_{Q}(s)$. We set

$$
Z_{Q}(s)=\sum_{\substack{x, y=-\infty \\ Q(x, y)>0 \\ 2 a x+(b-\sqrt{d}) y>0 \\ 1 \leq\left|\frac{2 a x+(b+\sqrt{d}) y}{2 a x+(b-\sqrt{d}) y}\right|<\varepsilon^{2}}}^{\infty} \frac{1}{Q(x, y)^{s}} .
$$

It is shown in Section 3 that the series in (12) converges for $s>1$ so that $Z_{Q}(s)$ is defined for $s>1$. Also in Section 3, it is shown that as $s \rightarrow 1^{+}$

$$
Z_{Q}(s)=\frac{\log \varepsilon(d)}{\sqrt{d}} \cdot \frac{1}{s-1}+C_{Q}+O(s-1)
$$

for an explicit constant $C_{Q}$ (see Theorem 2). We remark that Zagier [16: Theorems, pp. 166-167] has considered a different analogue of the Epstein 
zeta function, namely, the infinite series

$$
\sum_{x=1, y=0}^{\infty} \frac{1}{Q(x, y)^{s}}
$$

for an indefinite binary quadratic form $Q=(a, b, c)$ of discriminant $d>0$ with $a>0, b>0$ and $c>0$. Let $G \in G(d)$. Since

$$
\sum_{n=1}^{\infty} \frac{R_{G}(n, d)}{n^{s}}=\sum_{[Q] \in G} Z_{Q}(s),
$$

we obtain from (8), (13) and (14)

$$
\begin{aligned}
\frac{h(d) \log \varepsilon(d)}{2^{t(d)} \sqrt{d}} \cdot \frac{1}{s-1}+ & B(d)+\beta(d, G)+O(s-1) \\
& =\sum_{[Q] \in G}\left(\frac{\log \varepsilon(d)}{\sqrt{d}} \cdot \frac{1}{s-1}+C_{Q}+O(s-1)\right) \\
& =\frac{h(d) \log \varepsilon(d)}{2^{t(d)} \sqrt{d}} \cdot \frac{1}{s-1}+\sum_{[Q] \in G} C_{Q}+O(s-1),
\end{aligned}
$$

so that

$$
B(d)+\beta(d, G)=\sum_{[Q] \in G} C_{Q}
$$

The formula (15) provides an analogue of the Chowla-Selberg formula for genera in the case of positive discriminants. However the constant $B(d)$ contains the quantity $L^{\prime}(1, \Delta)$ (see $(98)$ and $(100)$ ), which is difficult to give explicitly (see Deninger [6]). Thus in Section 5 we eliminate $B(d)$ from $(15)$ to obtain a simpler formula. Let $G_{1}$ and $G_{2}$ be two genera of $G(d)$. Then, from (15), we obtain

$$
\beta\left(d, G_{1}\right)-\beta\left(d, G_{2}\right)=\sum_{[Q] \in G_{1}} C_{Q}-\sum_{[Q] \in G_{2}} C_{Q}
$$

Putting the explicit expressions for $\beta\left(d, G_{k}\right)(k=1,2)$ and $C_{Q}$ into (16), we obtain Theorem 5 .

By taking particular choices of the genera $G_{1}$ and $G_{2}$ in Theorem 5 , we are able to evaluate explicitly certain definite integrals. The nature of these integrals suggests that it would be difficult to evaluate them by conventional means, a view previously expressed by Chowla ([3: p. 372], [4: p. 1019]). These integrals are given in Theorems $6-10$. They include three integrals 
given by Herglotz [11: p. 14] as well as many new ones such as

$$
\int_{0}^{1} \frac{\tan ^{-1}\left(t^{3+\sqrt{8}}\right)}{1+t^{2}} d t=\frac{1}{16} \log 2 \log (3+\sqrt{8})
$$

and

$$
\begin{aligned}
\int_{0}^{1} \frac{\log \left(1+t^{13+\sqrt{168}}\right)}{1+t} d t= & \frac{\pi^{2}}{24}(13-\sqrt{672}) \\
& +\frac{1}{2} \log (1+\sqrt{2}) \log \left(\frac{5+\sqrt{21}}{2}\right) \\
& +\frac{1}{4} \log (2+\sqrt{3}) \log (15+\sqrt{224}) \\
& +\frac{1}{4} \log (5+\sqrt{24}) \log (8+\sqrt{63}) \\
& +\frac{1}{2} \log 2 \log \left(2(13+\sqrt{168})^{3 / 2}\right)
\end{aligned}
$$

(see Theorem 10).

2. Formula for $R_{G}(n, d)$. Proof of Theorem 1. In this section, up to and including Lemma 12, $d$ may be either positive or negative. From Lemma 13 on, and throughout the rest of the paper, $d$ is assumed to be positive.

The discriminants $-4,8,-8$ and $p^{*}=(-1)^{(p-1) / 2} p(p$ prime $>2)$, are called prime discriminants. The prime discriminants corresponding to the discriminant $d$ are the discriminants $p_{1}^{*}, \ldots, p_{t+1}^{*}$, together with $p_{t+2}^{*}$ if $d \equiv 0$ $(\bmod 32)$, where $t=t(d)$, given as follows:

- $d \equiv 1(\bmod 4)$ or $d \equiv 4(\bmod 16)$, $p_{1}<\ldots<p_{t+1}$ are the odd prime divisors of $d$.

- $d \equiv 12(\bmod 16)$ or $d \equiv 16(\bmod 32)$, $p_{1}<\ldots<p_{t}$ are the odd prime divisors of $d$ and $p_{t+1}^{*}=-4$.

- $d \equiv 8(\bmod 32)$, $p_{1}<\ldots<p_{t}$ are the odd prime divisors of $d$ and $p_{t+1}^{*}=8$.

- $d \equiv 24(\bmod 32)$, $p_{1}<\ldots<p_{t}$ are the odd prime divisors of $d$ and $p_{t+1}^{*}=-8$.

- $d \equiv 0(\bmod 32)$, $p_{1}<\ldots<p_{t-1}$ are the odd prime divisors of $d, p_{t}^{*}=-4, p_{t+1}^{*}=8$, $p_{t+2}^{*}=-8$.

Following Huard, Kaplan and Williams [13] we denote the set of prime discriminants corresponding to $d$ by $P(d)$. We denote the set of all products of pairwise coprime elements of $P(d)$ by $F(d)$. 
It is known that a fundamental discriminant $d$ can be written uniquely as a product of pairwise coprime prime discriminants and that any such product is a fundamental discriminant [15: Proposition 9]. It is easy to check that the prime discriminants occurring in such a decomposition are precisely the elements of $P(d)$. It is convenient at this point to note some properties of the set $F(d)$.

Lemma 1. (a) $F(d)=\left\{d_{1}: d_{1}\right.$ is a fundamental discriminant, $d_{1} \mid d$, and $d / d_{1}$ is a discriminant $\}$.

(b) For any positive integer $k, P(d) \subseteq P\left(d k^{2}\right)$ and $F(d) \subseteq F\left(d k^{2}\right)$. Also, $P(\Delta) \subseteq P(d), 1 \in F(d), \Delta \in F(d),|F(\bar{d})|=2^{t(d)+1}$, and

$$
|P(d)|= \begin{cases}t(d)+2 & \text { if } d \equiv 0(\bmod 32), \\ t(d)+1 & \text { otherwise. }\end{cases}
$$

(c) If $d_{1} \in F(d)$ then $f\left(d / d_{1}\right) \mid f(d)$.

(d) Let $m$ be a positive integer such that $m \mid f$. Let $d_{1} \in F\left(d / m^{2}\right)$. Then

$$
f\left(d / m^{2} d_{1}\right) \mid f / m \text { and } m \mid f\left(d / d_{1}\right) .
$$

(e) Let $m$ be a positive integer. Then

$$
m\left|f(d), d_{1} \in F\left(d / m^{2}\right) \Leftrightarrow d_{1} \in F(d), m\right| f\left(d / d_{1}\right) .
$$

Proof. (a), (b). These two parts of the lemma are given in [13: Lemma 2.1] for the case $d<0$. It is easy to check that they are also valid for $d>0$.

(c) As $d_{1} \in F(d)$, by (a) we see that $d_{1}$ and $d / d_{1}$ are discriminants with $d_{1} \cdot d / d_{1}=d$, so that by the properties given at the beginning of Section 1 , we have $f\left(d / d_{1}\right) \mid f(d)$.

(d) As $m$ is a positive integer such that $m \mid f$ we have $m^{2} \mid d, d / m^{2}$ is a discriminant, and $f\left(d / m^{2}\right)=f / m$. Further, as $d_{1} \in F\left(d / m^{2}\right), d_{1}$ is a fundamental discriminant such that $d_{1} \mid d / m^{2}$ and $d_{2}=\frac{d / m^{2}}{d_{1}}$ is a discriminant. From $d_{1} d_{2}=d / m^{2}$ we have $f\left(d_{2}\right) \mid f\left(d / m^{2}\right)$, that is, $f\left(d / m^{2} d_{1}\right) \mid f / m$, as asserted. Also $d / d_{1}=d_{2} m^{2}$ so that

$$
f\left(d / d_{1}\right)=f\left(d_{2} m^{2}\right)=f\left(d_{2}\right) m
$$

that is, $m \mid f\left(d / d_{1}\right)$.

(e) Suppose first that $m \mid f(d)$ and $d_{1} \in F\left(d / m^{2}\right)$. Then $d_{1} \in F(d)$ by (b) and $m \mid f\left(d / d_{1}\right)$ by (d). Hence we have shown that

$$
m\left|f(d), d_{1} \in F\left(d / m^{2}\right) \Rightarrow d_{1} \in F(d), m\right| f\left(d / d_{1}\right) .
$$

Now suppose that $d_{1} \in F(d)$ and $m \mid f\left(d / d_{1}\right)$. By (a), $d_{1}$ is a fundamental discriminant such that $d_{1} \mid d$ and $d / d_{1}$ is a discriminant. As $m \mid f\left(d / d_{1}\right)$, from

$$
d / d_{1}=\Delta\left(d / d_{1}\right) f\left(d / d_{1}\right)^{2},
$$


we deduce that $d_{1} \mid d / m^{2}$ and $d / d_{1} m^{2}$ is a discriminant so that $d_{1} \in F\left(d / m^{2}\right)$ by (a). As $m \mid f\left(d / d_{1}\right)$ we have $m \mid f(d)$ by (c) so we have shown that

$$
d_{1} \in F(d), m\left|f\left(d / d_{1}\right) \Rightarrow m\right| f(d), d_{1} \in F\left(d / m^{2}\right) .
$$

This completes the proof of Lemma 1.

Next, we recall the basic properties of generic characters (see for example [1: Chapter 4]). Let $p^{*} \in P(d)$ and $K \in H(d)$. For any positive integer $k$ coprime with $p^{*}$, which is represented by $K$, it is known that $\left(\frac{p^{*}}{k}\right)$ has the same value, so we can set

$$
\gamma_{p^{*}}(K)=\left(\frac{p^{*}}{k}\right)= \pm 1
$$

Let $G \in G(d)$. It is known that for any $K \in G, \gamma_{p^{*}}(K)$ has the same value, so we can set $\gamma_{p^{*}}(G)=\gamma_{p^{*}}(K)$. Also,

$$
\gamma_{p^{*}}\left(G_{1} G_{2}\right)=\gamma_{p^{*}}\left(G_{1}\right) \gamma_{p^{*}}\left(G_{2}\right),
$$

for $G_{1}, G_{2} \in G(d)$. An important result of genus theory is the following product formula due to Gauss (see for example [9: equation (9)]).

Lemma 2. (a) If $G \in G(d)$ then with $\Delta=\Delta(d)$,

$$
\prod_{p^{*} \in P(\Delta)} \gamma_{p^{*}}(G)=1
$$

together with

$$
\gamma_{-4}(G) \gamma_{8}(G) \gamma_{-8}(G)=1 \quad \text { if } d \equiv 0(\bmod 32) .
$$

(b) Moreover, if $\delta_{p^{*}}= \pm 1$ for each $p^{*} \in P(d)$ and $\prod_{p^{*} \in P(\Delta)} \delta_{p^{*}}=1$, together with

$$
\delta_{-4} \delta_{8} \delta_{-8}=1 \quad \text { if } d \equiv 0(\bmod 32),
$$

then there exists a unique genus $G \in G(d)$ with

$$
\gamma_{p^{*}}(G)=\delta_{p^{*}} \quad \text { for each } p^{*} \in P(d) .
$$

For $d_{1} \in F(d)$, we set

$$
\gamma_{d_{1}}(G)=\prod_{p^{*} \in P\left(d_{1}\right)} \gamma_{p^{*}}(G)= \pm 1 .
$$

We let $v_{p}(n)$ denote the exponent of the highest power of the prime $p$ dividing $n$. Following [13] we define for all discriminants $d$ the derived genus $G_{m} \in G\left(d /(m, f)^{2}\right)$ of $G \in G(d)$, where $m$ is a positive integer all of whose prime factors $p$ divide $d$ and satisfy

$$
p \nmid \Delta \Rightarrow v_{p}(m) \leq v_{p}(f) .
$$

We begin with the case when $m$ is a prime. 
Lemma 3. Let $p$ be a prime with $p \mid d$, and let $G \in G(d)$. Then there is a unique genus

$$
G_{p} \in \begin{cases}G\left(d / p^{2}\right) & \text { if } p \mid f, \\ G(d) & \text { if } p \nmid f,\end{cases}
$$

such that in the case $p \mid f$,

$$
\gamma_{q^{*}}\left(G_{p}\right)=\gamma_{q^{*}}(G) \quad \text { for all } q^{*} \in P\left(d / p^{2}\right),
$$

and in the case $p \nmid f$, for every $q^{*} \in P(d)$ with $p \nmid q^{*}$,

$$
\gamma_{q^{*}}\left(G_{p}\right)=\left(\frac{q^{*}}{p}\right) \gamma_{q^{*}}(G),
$$

and, for the unique $q^{*} \in P(d)$ with $p \mid q^{*}$,

$$
\gamma_{q^{*}}\left(G_{p}\right)=\left(\frac{d / q^{*}}{p}\right) \gamma_{q^{*}}(G)=\left(\frac{\Delta / q^{*}}{p}\right) \gamma_{q^{*}}(G) .
$$

Proof. The proof is exactly the same as the proof of Proposition 3.1 in [13] for the case $d<0$.

Next, we define $G_{p^{i}}$ for $p \mid d$ and $i \geq 0$. We set $G_{1}=G$. By (25), we define successively

$$
G_{p^{i}}=\left(G_{p^{i-1}}\right)_{p} \in G\left(d / p^{2 i}\right) \quad \text { for } i=1, \ldots, v_{p}(f) .
$$

If in addition $p \mid \Delta$, as $p \nmid f / p^{v_{p}(f)}$, we define successively

$$
G_{p^{i}}=\left(G_{p^{i-1}}\right)_{p} \in G\left(d / p^{2 v_{p}(f)}\right), \quad i=1+v_{p}(f), \ldots
$$

Thus, for any $p \mid d$, we have defined $G_{p^{i}} \in G\left(d /\left(p^{i}, f\right)^{2}\right)$ for any $i \geq 0$ if $p \mid \Delta$ and for $0 \leq i \leq v_{p}(f)$ if $p \nmid \Delta$. For $m=p_{1}^{\alpha_{1}} \ldots p_{r}^{\alpha_{r}}$ satisfying (24), we define

$$
G_{m}=\left(\ldots\left(\left(G_{p_{1}^{\alpha_{1}}}\right) p_{2}^{\alpha_{2}}\right) \ldots\right)_{p_{r}^{\alpha_{r}}} \in G\left(d /(m, f)^{2}\right) .
$$

It is easily checked that the order of the $p_{i}$ 's does not matter.

Lemma 4. (a) Let $p$ be a prime with $p \mid d$. Let $d_{1} \in F\left(d /(p, f)^{2}\right)$. Then, for any $G \in G(d)$, we have

$$
\gamma_{d_{1}}\left(G_{p}\right)= \begin{cases}\gamma_{d_{1}}(G) & \text { if } p \mid f, \\ \left(\frac{d_{1}}{p}\right) \gamma_{d_{1}}(G) & \text { if } p \nmid f, p \nmid d_{1}, \\ \left(\frac{d / d_{1}}{p}\right) \gamma_{d_{1}}(G) & \text { if } p \nmid f, p \mid d_{1} .\end{cases}
$$

(b) If $m$ is a positive integer with $m \mid f, G \in G(d)$ and $d_{1} \in F\left(d / m^{2}\right)$ then $\gamma_{d_{1}}\left(G_{m}\right)=\gamma_{d_{1}}(G)$.

Proof. The proof is exactly the same as the proof of Lemma 3.1 in [13] for the case $d<0$. 

if

Following [13] we define a prime $p$ to be a null prime relative to $n$ and $d$

$$
v_{p}(n) \equiv 1(\bmod 2), \quad v_{p}(n)<2 v_{p}(f) .
$$

We denote the set of all such null primes by $\operatorname{Null}(n, d)$.

Lemma 5. If $\operatorname{Null}(n, d) \neq \emptyset$, then $R_{K}(n, d)=0$ for each $K \in H(d)$.

Proof. The proof is exactly the same as the proof of Proposition 4.1 in [13] for the case $d<0$.

Next, as in [13: Section 4], we introduce three positive integers $M, U$ and $Q$ :

$$
\begin{gathered}
M=M(n, d) \text { is the largest integer such that } M^{2} \mid n \text { and } M \mid f, \\
U=U(n, d)=\prod_{\substack{p \mid d \\
p \nmid f}} p^{v_{p}(n)}, \\
Q=Q(n, d)=U\left(n / M^{2}, d / M^{2}\right)=\prod_{\substack{p \mid d / M^{2} \\
p \nmid f / M}} p^{v_{p}\left(n / M^{2}\right)} .
\end{gathered}
$$

LEMMA 6. (a) If $\operatorname{Null}(n, d)=\emptyset$ then

$$
\left(n / M^{2}, f / M\right)=1 \quad \text { and } \quad\left(n / M^{2} Q, d / M^{2}\right)=1 .
$$

(b) $(n, f)=1$ if and only if $\operatorname{Null}(n, d)=\emptyset$ and $M=1$.

Proof. The proof is the same as the proof of Lemma 4.1 in [13].

For $d_{1} \in F(d)$ and $(n, f)=1$, we set

$$
S\left(n, d_{1}, d / d_{1}\right)=\sum_{\mu \nu=n}\left(\frac{d_{1}}{\mu}\right)\left(\frac{d / d_{1}}{\nu}\right)
$$

where $\mu$ and $\nu$ run through all positive integers with $\mu \nu=n$.

LEMMA 7. Let $(n, f)=1$ and let $p$ be a prime dividing both $n$ and $d$. Then, for $G \in G(d)$, we have

$$
\sum_{d_{1} \in F(d)} \gamma_{d_{1}}(G) S\left(n, d_{1}, d / d_{1}\right)=\sum_{d_{1} \in F(d)} \gamma_{d_{1}}\left(G_{p}\right) S\left(n / p, d_{1}, d / d_{1}\right) .
$$

Proof. The proof is the same as that of Lemma 5.1 in [13].

Lemma 8. Let $(n, f)=1$. Then, for $G \in G(d)$, we have

$$
\sum_{d_{1} \in F(d)} \gamma_{d_{1}}(G) S\left(n, d_{1}, d / d_{1}\right)=\sum_{d_{1} \in F(d)} \gamma_{d_{1}}\left(G_{U}\right) S\left(n / U, d_{1}, d / d_{1}\right),
$$

where $U$ is defined in (32). 
Proof. This follows by repeatedly applying Lemma 7 to all the primes dividing the integer $U$.

Lemma 9. Let $p$ be a prime with $p \mid d, p \nmid f$. Let $K \in H(d)$. Then

(a) $K$ contains a form $(a, b, c p)$ with $p \nmid a c, p \mid b$;

(b) the mapping $\phi_{p}: H(d) \rightarrow H(d)$ given by $\phi_{p}([a, b, c p])=[a p, b, c]$, where $(a, b, c p)$ is as in (a), is a bijection;

(c) if $G \in G(d)$ and $K \in G$ then $\phi_{p}(K) \in G_{p}$.

Proof. The proof is the same as the proof of Lemma 7.1 in [13].

Lemma 10. Let $p$ be a prime with $p|n, p| d$ and $p \nmid f$. Then, for $K \in$ $H(d)$, we have $R_{K}(n, d)=R_{\phi_{p}(K)}(n / p, d)$.

Proof. Let $(a, b, c p) \in K$ with $p \nmid a c, p \mid b$. Then $(a p, b, c) \in \phi_{p}(K)$. We set

$$
\begin{aligned}
& S=\left\{(x, y) \in \mathbb{Z}^{2}: a x^{2}+b x y+c p y^{2}=n,(x, y) \text { primary }\right\}, \\
& T=\left\{(X, Y) \in \mathbb{Z}^{2}: a p X^{2}+b X Y+c Y^{2}=n / p,(X, Y) \text { primary }\right\} .
\end{aligned}
$$

It is easily checked that $(X, Y) \mapsto(p X, Y)$ is a bijection from $T$ to $S$.

Lemma 11. Let $p$ be a prime with $p|n, p| d$ and $p \nmid f$. Then, for $G \in$ $G(d)$, we have $R_{G}(n, d)=R_{G_{p}}(n / p, d)$.

Proof. We have

$$
\begin{aligned}
R_{G}(n, d) & =\sum_{K \in G} R_{K}(n, d)=\sum_{K \in G} R_{\phi_{p}(K)}(n / p, d) \\
& =\sum_{K^{\prime} \in G_{p}} R_{K^{\prime}}(n / p, d)=R_{G_{p}}(n / p, d),
\end{aligned}
$$

by Lemmas 9 and 10 .

We are now ready to prove our first reduction formula.

Proposition 1. For $G \in G(d)$, we have

$$
R_{G}(n, d)=R_{G_{U}}(n / U, d)
$$

where $U=U(n, d)$ is defined in (32).

Proof. This follows from Lemma 11 by repeatedly applying it to all the primes dividing $U$.

Lemma 12. Let $p \mid f, K \in H(d)$ and let $l$ be a positive integer. Then

(a) $K$ contains a form $(a, b, c)$ with $p\left|b, p^{2}\right| c$ and $(a, p l)=1$;

(b) the mapping $\theta_{p}: H(d) \rightarrow H\left(d / p^{2}\right)$ given by $\theta_{p}([a, b, c])=\left[a, b / p, c / p^{2}\right]$, where $(a, b, c)$ is as in (a), is a surjective homomorphism;

(c) if $G \in G(d)$ and $K \in G$ then $\theta_{p}(K) \in G_{p}$;

(d) the mapping $\widehat{\theta}_{p}: G(d) \rightarrow G\left(d / p^{2}\right)$ given by $\widehat{\theta}_{p}(G)=G_{p}$ is a surjective homomorphism. 
Proof. The proof is exactly the same as that of Lemma 6.1 in [13].

From this point on we assume that $d>0$. Set

Lemma 13. Let $d>0,[a, b, c] \in H(d)$ and let $m$ be a positive integer.

$$
\begin{gathered}
S=\left\{(x, y) \in \mathbb{Z}^{2}: a x^{2}+b x y+c y^{2}=n, 2 a x+(b-\sqrt{d}) y>0,\right. \\
\left.1 \leq\left|\frac{2 a x+(b+\sqrt{d}) y}{2 a x+(b-\sqrt{d}) y}\right|<\varepsilon^{2}\right\}, \\
T=\left\{(X, Y) \in \mathbb{Z}^{2}: a X^{2}+b X Y+c Y^{2}=n, 2 a X+(b-\sqrt{d}) Y>0,\right. \\
\left.\varepsilon^{2 m} \leq\left|\frac{2 a X+(b+\sqrt{d}) Y}{2 a X+(b-\sqrt{d}) Y}\right|<\varepsilon^{2 m+2}\right\},
\end{gathered}
$$

where $\varepsilon=\varepsilon(d)$ is defined in (3). Then $\operatorname{card} S=\operatorname{card} T$.

Proof. Let

$$
\varepsilon^{\prime}=\frac{1}{\varepsilon}=\frac{x_{0}-y_{0} \sqrt{d}}{2} \text { and } \varepsilon^{m}=\frac{t+u \sqrt{d}}{2},
$$

where $t$ and $u$ are rational numbers. Then

$$
\varepsilon^{\prime m}=\frac{t-u \sqrt{d}}{2} .
$$

Adding we obtain $t=\varepsilon^{m}+\varepsilon^{\prime m}$. As $\varepsilon$ is an algebraic integer, so are $\varepsilon^{\prime}, \varepsilon^{m}$ and $\varepsilon^{\prime m}$. Hence $t$ is an algebraic integer and thus, as it is rational, it must be an integer. Similarly

$$
u=y_{0} \frac{\varepsilon^{m}-\varepsilon^{\prime m}}{\varepsilon-\varepsilon^{\prime}}
$$

is an algebraic integer, and thus as it is rational, it must be an integer. Finally, as $\varepsilon \varepsilon^{\prime}=1$, we deduce that the integers $t$ and $u$ satisfy $t^{2}-d u^{2}=4$.

We define a map from $S$ to $T$ by $(x, y) \mapsto(X, Y)$, where

$$
\left(\begin{array}{l}
X \\
Y
\end{array}\right)=\left(\begin{array}{cc}
(t-b u) / 2 & -c u \\
a u & (t+b u) / 2
\end{array}\right)\left(\begin{array}{l}
x \\
y
\end{array}\right) .
$$

Easy calculations show that

$$
\begin{gathered}
a x^{2}+b x y+c y^{2}=a X^{2}+b X Y+c Y^{2}, \\
2 a X+(b+\sqrt{d}) Y=\varepsilon^{m}(2 a x+(b+\sqrt{d}) y) .
\end{gathered}
$$

Hence

$$
2 a X+(b-\sqrt{d}) Y=\varepsilon^{\prime m}(2 a x+(b-\sqrt{d}) y) .
$$

It is now easily verified that the map $(x, y) \mapsto(X, Y)$ is a bijection. 
Lemma 14. Let $d>0$. Let $p$ be a prime with $p \mid M$, where $M$ is defined in (31). Then, for any $K \in H(d)$, we have

$$
R_{K}(n, d)=\frac{\log \varepsilon(d)}{\log \varepsilon\left(d / p^{2}\right)} R_{\theta_{p}(K)}\left(n / p^{2}, d / p^{2}\right) .
$$

Proof. We begin by choosing $(a, b, c) \in K$ with $p \nmid a, p \mid b$ and $p^{2} \mid c$ so that $\theta_{p}(K)=\left[a, b / p, c / p^{2}\right]$. Then we set

$$
\begin{gathered}
S=\left\{(x, y) \in \mathbb{Z}^{2}: a x^{2}+b x y+c y^{2}=n, 2 a x+(b-\sqrt{d}) y>0,\right. \\
\left.1 \leq\left|\frac{2 a x+(b+\sqrt{d}) y}{2 a x+(b-\sqrt{d}) y}\right|<\varepsilon(d)^{2}\right\}, \\
T=\left\{(X, Y) \in \mathbb{Z}^{2}: \frac{n}{p^{2}}=a X^{2}+\frac{b X Y}{p}+\frac{c Y^{2}}{p^{2}}, 2 a X+(b-\sqrt{d}) \frac{Y}{p}>0,\right. \\
\left.1 \leq\left|\frac{2 a X+(b+\sqrt{d}) Y / p}{2 a X+(b-\sqrt{d}) Y / p}\right|<\varepsilon(d)^{2}\right\}, \\
V=\left\{(X, Y) \in \mathbb{Z}^{2}: \frac{n}{p^{2}}=a X^{2}+\frac{b X Y}{p}+\frac{c Y^{2}}{p^{2}}, 2 a X+(b-\sqrt{d}) \frac{Y}{p}>0,\right. \\
\left.1 \leq\left|\frac{2 a X+(b+\sqrt{d}) Y / p}{2 a X+(b-\sqrt{d}) Y / p}\right|<\varepsilon\left(d / p^{2}\right)^{2}\right\} .
\end{gathered}
$$

All solutions in integers to $x^{2}-d y^{2}=4$ are given by

$$
\frac{x+y \sqrt{d}}{2}= \pm \varepsilon^{m}, \quad m \in \mathbb{Z},
$$

(see for example [12: Theorem 4.4, p. 281]). As $x=x_{0}, y=p y_{0}$ is an integral solution of $x^{2}-\left(d / p^{2}\right) y^{2}=4$, we have

$$
\varepsilon(d)=\frac{x_{0}+y_{0} \sqrt{d}}{2}=\frac{x+(y / p) \sqrt{d}}{2}= \pm \varepsilon\left(d / p^{2}\right)^{m}
$$

for some $m \in \mathbb{Z}$. Moreover as $\varepsilon(d)$ and $\varepsilon\left(d / p^{2}\right)$ are both $>1$ we have $\varepsilon(d)=\varepsilon\left(d / p^{2}\right)^{m}$ and $m$ is a positive integer. The map from $T$ to $S$ given by $(X, Y) \mapsto(p X, Y)$ is easily seen to be a bijection. Thus

$$
R_{K}(n, d)=\operatorname{card} S=\operatorname{card} T=m \operatorname{card} V=\frac{\log \varepsilon(d)}{\log \varepsilon\left(d / p^{2}\right)} R_{\theta_{p}(K)}\left(n / p^{2}, d / p^{2}\right),
$$

by Lemma 13 .

Our next lemma is the analogue of [13: Lemma 6.3] for the case $d>0$. As the proof in [13] is fairly brief, we provide all the details here. 
Lemma 15. Let $d>0$. Let $p$ be a prime with $p \mid M$. Then, for $G \in G(d)$, we have

$$
R_{G}(n, d)=\frac{\log \varepsilon(d)}{\log \varepsilon\left(d / p^{2}\right)} \cdot \frac{h(d) / 2^{t(d)}}{h\left(d / p^{2}\right) / 2^{t\left(d / p^{2}\right)}} R_{G_{p}}\left(n / p^{2}, d / p^{2}\right) .
$$

Proof. Let $G \in G(d)$ and $L \in G_{p}$. As $G \in G(d)=H(d) / H^{2}(d)$ there exists a class $K_{1} \in H(d)$ such that $G=K_{1} H^{2}(d)$. Thus $K_{1} \in G$ and so $\theta_{p}\left(K_{1}\right) \in G_{p}$. Hence

$$
G_{p}=\theta_{p}\left(K_{1}\right) H^{2}\left(d / p^{2}\right) .
$$

As $L \in G_{p}$ there exists $L_{1} \in H\left(d / p^{2}\right)$ such that $L=\theta_{p}\left(K_{1}\right) L_{1}^{2}$. Further, as the homomorphism $\theta_{p}: H(d) \rightarrow H\left(d / p^{2}\right)$ is surjective, there exists a class $K_{2} \in H(d)$ such that $\theta_{p}\left(K_{2}\right)=L_{1}$. Set $A=K_{1} K_{2}^{2}$ so that $A \in G$ and

$$
\theta_{p}(A)=\theta_{p}\left(K_{1} K_{2}^{2}\right)=\theta_{p}\left(K_{1}\right) \theta_{p}\left(K_{2}\right)^{2}=\theta_{p}\left(K_{1}\right) L_{1}^{2}=L .
$$

Also $G=A H^{2}(d)$. Set

$$
N_{G}(L)=\sum_{\substack{K \in G \\ \theta_{p}(K)=L}} 1
$$

Then

$$
\begin{aligned}
N_{G}(L) & =\left|\left\{K \in G: \theta_{p}(K)=L\right\}\right|=\left|\left\{K \in H(d): \theta_{p}(K)=L\right\} \cap G\right| \\
& =\left|A \operatorname{ker} \theta_{p} \cap G\right|=\left|A \operatorname{ker} \theta_{p} \cap A H^{2}(d)\right| \\
& =\left|A\left(\operatorname{ker} \theta_{p} \cap H^{2}(d)\right)\right|=\left|\operatorname{ker} \theta_{p} \cap H^{2}(d)\right|,
\end{aligned}
$$

so that $N_{G}(L)$ is independent of $G$ and $L$. Hence

$$
\begin{aligned}
|G| & =\sum_{K \in G} 1=\sum_{\substack{K \in G \\
\theta_{p}(K) \in G_{p}}} 1=\sum_{L \in G_{p}} \sum_{\substack{K \in G \\
\theta_{p}(K)=L}} 1 \\
& =\sum_{L \in G_{p}} N_{G}(L)=N_{G}(L) \sum_{L \in G_{p}} 1=N_{G}(L)\left|G_{p}\right|,
\end{aligned}
$$

so that

$$
N_{G}(L)=\frac{|G|}{\left|G_{p}\right|}=\frac{h(d) / 2^{t(d)}}{h\left(d / p^{2}\right) / 2^{t\left(d / p^{2}\right)}} .
$$

Hence we have

$$
\begin{aligned}
R_{G}(n, d) & =\sum_{K \in G} R_{K}(n, d) \\
& =\frac{\log \varepsilon(d)}{\log \varepsilon\left(d / p^{2}\right)} \sum_{K \in G} R_{\theta_{p}(K)}\left(n / p^{2}, d / p^{2}\right) \quad \text { (by Lemma 14) } \\
& =\frac{\log \varepsilon(d)}{\log \varepsilon\left(d / p^{2}\right)} \sum_{L \in G_{p}} \sum_{\substack{K \in G \\
\theta_{p}(K)=L}} R_{\theta_{p}(K)}\left(n / p^{2}, d / p^{2}\right)
\end{aligned}
$$




$$
\begin{aligned}
& =\frac{\log \varepsilon(d)}{\log \varepsilon\left(d / p^{2}\right)} \sum_{L \in G_{p}} \sum_{\substack{K \in G \\
\theta_{p}(K)=L}} R_{L}\left(n / p^{2}, d / p^{2}\right) \\
& =\frac{\log \varepsilon(d)}{\log \varepsilon\left(d / p^{2}\right)} \sum_{L \in G_{p}} R_{L}\left(n / p^{2}, d / p^{2}\right) \sum_{\substack{K \in G \\
\theta_{p}(K)=L}} 1 \\
& =\frac{\log \varepsilon(d)}{\log \varepsilon\left(d / p^{2}\right)} \sum_{L \in G_{p}} R_{L}\left(n / p^{2}, d / p^{2}\right) N_{G}(L) \\
& =\frac{\log \varepsilon(d)}{\log \varepsilon\left(d / p^{2}\right)} \cdot \frac{h(d) / 2^{t(d)}}{h\left(d / p^{2}\right) / 2^{t\left(d / p^{2}\right)}} \sum_{L \in G_{p}} R_{L}\left(n / p^{2}, d / p^{2}\right) \\
& =\frac{\log \varepsilon(d)}{\log \varepsilon\left(d / p^{2}\right)} \cdot \frac{h(d) / 2^{t(d)}}{h\left(d / p^{2}\right) / 2^{t\left(d / p^{2}\right)}} R_{G_{p}}\left(n / p^{2}, d / p^{2}\right),
\end{aligned}
$$

as asserted.

We now give our second reduction formula.

Proposition 2. For $G \in G(d), d>0$, we have

$$
R_{G}(n, d)=\frac{1}{2^{t(d)-t\left(d / M^{2}\right)}} \cdot \frac{\log \varepsilon(d)}{\log \varepsilon\left(d / M^{2}\right)} \cdot \frac{h(d)}{h\left(d / M^{2}\right)} R_{G_{M}}\left(n / M^{2}, d / M^{2}\right) .
$$

Proof. This follows from Lemma 15 by applying it to all the primes dividing the integer $M$.

We now set

$$
N(n, d)=\sum_{K \in H(d)} R_{K}(n, d) .
$$

For $d>0$ Dirichlet (see for example [12: Theorem 4.1, p. 307]) has shown that

$$
N(n, d)=\sum_{\nu \mid n}\left(\frac{d}{\nu}\right) \quad \text { if }(n, d)=1 .
$$

Following the proof of Theorem 8.3 in [13] and using Dirichlet's result, we obtain

Proposition 3. Let $d>0$. If $(n, d)=1$ and $G \in G(d)$ then

$$
R_{G}(n, d)=\frac{1}{2^{t(d)+1}} \sum_{d_{1} \in F(d)} \gamma_{d_{1}}(G) S\left(n, d_{1}, d / d_{1}\right) .
$$

We are now ready to prove Theorem 1. 
Theorem 1. Let $G \in G(d), d>0$. If $\operatorname{Null}(n, d)=\emptyset$ then

$$
\begin{aligned}
R_{G}(n, d)= & \frac{\log \varepsilon(d)}{\log \varepsilon\left(d / M^{2}\right)} \cdot \frac{h(d)}{h\left(d / M^{2}\right)} \cdot \frac{1}{2^{t(d)+1}} \\
& \times \sum_{d_{1} \in F\left(d / M^{2}\right)} \gamma_{d_{1}}(G) S\left(n / M^{2}, d_{1}, d / M^{2} d_{1}\right) .
\end{aligned}
$$

If $\operatorname{Null}(n, d) \neq \emptyset$ then $R_{G}(n, d)=0$.

Proof. Suppose $\operatorname{Null}(n, d)=\emptyset$. By Propositions 1 and 2, we have

$$
\begin{aligned}
R_{G}(n, d) & =\frac{1}{2^{t(d)-t\left(d / M^{2}\right)}} \cdot \frac{\log \varepsilon(d)}{\log \varepsilon\left(d / M^{2}\right)} \cdot \frac{h(d)}{h\left(d / M^{2}\right)} R_{G_{M}}\left(n / M^{2}, d / M^{2}\right) \\
& =\frac{1}{2^{t(d)-t\left(d / M^{2}\right)}} \cdot \frac{\log \varepsilon(d)}{\log \varepsilon\left(d / M^{2}\right)} \cdot \frac{h(d)}{h\left(d / M^{2}\right)} R_{G_{M Q}}\left(n / M^{2} Q, d / M^{2}\right)
\end{aligned}
$$

as $U\left(n / M^{2}, d / M^{2}\right)=Q$ by (33). By Lemma 6 (a) we have

$$
\left(\frac{n}{M^{2} Q}, \frac{d}{M^{2}}\right)=1 \quad \text { and } \quad\left(\frac{n}{M^{2}}, \frac{f}{M}\right)=1,
$$

so that, by Proposition 3, Lemma 8 and Lemma 4(b), we have

$$
\begin{aligned}
R_{G}(n, d)= & \frac{1}{2^{t(d)-t\left(d / M^{2}\right)}} \cdot \frac{\log \varepsilon(d)}{\log \varepsilon\left(d / M^{2}\right)} \cdot \frac{h(d)}{h\left(d / M^{2}\right)} \cdot \frac{1}{2^{t\left(d / M^{2}\right)+1}} \\
& \times \sum_{d_{1} \in F\left(d / M^{2}\right)} \gamma_{d_{1}}\left(G_{M Q}\right) S\left(n / M^{2} Q, d_{1}, d / M^{2} d_{1}\right) \\
= & \frac{1}{2^{t(d)+1}} \cdot \frac{\log \varepsilon(d)}{\log \varepsilon\left(d / M^{2}\right)} \cdot \frac{h(d)}{h\left(d / M^{2}\right)} \\
& \times \sum_{d_{1} \in F\left(d / M^{2}\right)} \gamma_{d_{1}}\left(G_{M}\right) S\left(n / M^{2}, d_{1}, d / M^{2} d_{1}\right) \\
= & \frac{1}{2^{t(d)+1}} \cdot \frac{\log \varepsilon(d)}{\log \varepsilon\left(d / M^{2}\right)} \cdot \frac{h(d)}{h\left(d / M^{2}\right)} \\
& \times \sum_{d_{1} \in F\left(d / M^{2}\right)} \gamma_{d_{1}}(G) S\left(n / M^{2}, d_{1}, d / M^{2} d_{1}\right) .
\end{aligned}
$$

The second assertion of Theorem 1 follows from Lemma 5.

3. The restricted Epstein zeta function $Z_{Q}(s)$. Proof of Theorem 2. Let $a, b$ and $c$ be integers with $a>0, \operatorname{gcd}(a, b, c)=1$ and $b^{2}-4 a c=d$, where $d$ is a positive nonsquare discriminant. We set $Q(x, y)=$ $a x^{2}+b x y+c y^{2}$ so that $Q$ is an indefinite, primitive, integral, binary quadratic form of discriminant $d$. Let $\varepsilon=\varepsilon(d)$ be given by (3). For $s>1$, we define 
the restricted Epstein zeta function $Z_{Q}(s)$ by

$$
Z_{Q}(s)=\sum_{\substack{x, y=-\infty \\ Q(x, y)>0 \\ 2 a x+(b-\sqrt{d}) y>0 \\ 1 \leq\left|\frac{2 a x+(b+\sqrt{d}) y}{2 a x+(b-\sqrt{d}) y}\right|<\varepsilon^{2}}}^{\infty} \frac{1}{Q(x, y)^{s}} .
$$

We begin by showing that the series in (36) defining $Z_{Q}(s)$ converges for $s>1$. To do this, we examine the three parts of the series (36) corresponding to $y=0, y>0$ and $y<0$, and show that each converges for $s>1$.

The part corresponding to $y=0$ is clearly

$$
\sum_{x=1}^{\infty} \frac{1}{Q(x, 0)^{s}}=\sum_{x=1}^{\infty} \frac{1}{\left(a x^{2}\right)^{s}}=a^{-s} \zeta(2 s)
$$

for $s>1 / 2$.

For $y>0$ we show that the conditions in the definition of $Z_{Q}(s)$ are satisfied if and only if $2 a x>\lambda y$, where

$$
\lambda=-b+\sqrt{d}+2 \sqrt{d} /\left(\varepsilon^{2}-1\right) .
$$

Set

$$
E=2 a x+(b+\sqrt{d}) y, \quad E^{\prime}=2 a x+(b-\sqrt{d}) y .
$$

The summation conditions are

$$
E E^{\prime}>0, \quad E^{\prime}>0, \quad 1 \leq\left|E / E^{\prime}\right|<\varepsilon^{2},
$$

which are equivalent to

$$
E>0, \quad E^{\prime}>0, \quad E^{\prime} \leq E<\varepsilon^{2} E^{\prime} .
$$

For $y>0$ we have $E>E^{\prime}$ so these conditions are equivalent to

$$
E^{\prime}>0, \quad E<\varepsilon^{2} E^{\prime} .
$$

The second of these inequalities is equivalent to (as $\varepsilon>1$ )

$$
2 a x>\left(-b+\sqrt{d}+\frac{2 \sqrt{d}}{\varepsilon^{2}-1}\right) y .
$$

Moreover if this inequality holds then $2 a x>(-b+\sqrt{d}) y$ so that $E^{\prime}>0$. Hence the part corresponding to $y>0$ is

$$
\sum_{y=1}^{\infty} \sum_{x>\lambda_{1} y} \frac{1}{Q(x, y)^{s}}
$$

where

$$
\lambda_{1}=\lambda /(2 a)
$$


If $y<0$, a short calculation similar to the above shows that the conditions in the definition of $Z_{Q}(s)$ are never satisfied. Thus we must examine the convergence of

$$
\sum_{y=1}^{\infty} \sum_{x>\lambda_{1} y} \frac{1}{Q(x, y)^{s}}=\sum_{y=1}^{\infty} y^{-2 s} \sum_{x>\lambda_{1} y} Q\left(x y^{-1}, 1\right)^{-s} .
$$

To evaluate the inner sum in (41), we apply the Euler-Maclaurin summation formula. For $s>1 / 2, y>0$, we obtain

$$
\begin{aligned}
\sum_{x>\lambda_{1} y} Q\left(x y^{-1}, 1\right)^{-s} & =P\left(\lambda_{1} y\right) Q\left(\lambda_{1}, 1\right)^{-s}+\int_{\lambda_{1} y}^{\infty} Q\left(x y^{-1}, 1\right)^{-s} d x \\
& +\int_{\lambda_{1} y}^{\infty}(-s) Q\left(x y^{-1}, 1\right)^{-s-1}\left(2 a x y^{-2}+b y^{-1}\right) P(x) d x \\
= & y \int_{\lambda_{1}}^{\infty} Q(t, 1)^{-s} d t-s \int_{\lambda_{1}}^{\infty} Q(t, 1)^{-s-1}(2 a t+b) P(t y) d t \\
& +P\left(\lambda_{1} y\right) Q\left(\lambda_{1}, 1\right)^{-s},
\end{aligned}
$$

where $P(x)=x-[x]-1 / 2$. Thus, for $s>1$, we have

$$
\begin{aligned}
\sum_{y=1}^{\infty} y^{-2 s} \sum_{x>\lambda_{1} y} Q\left(x y^{-1}, 1\right)^{-s} & \\
= & \zeta(2 s-1) \int_{\lambda_{1}}^{\infty} Q(t, 1)^{-s} d t+Q\left(\lambda_{1}, 1\right)^{-s} \sum_{y=1}^{\infty} \frac{P\left(\lambda_{1} y\right)}{y^{2 s}} \\
& -s \sum_{y=1}^{\infty} y^{-2 s} \int_{\lambda_{1}}^{\infty} \frac{(2 a t+b) P(t y)}{Q(t, 1)^{s+1}} d t .
\end{aligned}
$$

This shows that

$$
\sum_{y=1}^{\infty} y^{-2 s} \sum_{x>\lambda_{1} y} Q\left(x y^{-1}, 1\right)^{-s},
$$

and thus the original series for $Z_{Q}(s)$ converges for $s>1$. Putting together (36), (37) and (43), we obtain

LeMma 16. For $s>1$, we have

$$
\begin{aligned}
Z_{Q}(s)= & a^{-s} \zeta(2 s)+\zeta(2 s-1) \int_{\lambda_{1}}^{\infty} Q(t, 1)^{-s} d t+Q\left(\lambda_{1}, 1\right)^{-s} \sum_{y=1}^{\infty} \frac{P\left(\lambda_{1} y\right)}{y^{2 s}} \\
& -s \sum_{y=1}^{\infty} y^{-2 s} \int_{\lambda_{1}}^{\infty} \frac{(2 a t+b) P(t y)}{Q(t, 1)^{s+1}} d t .
\end{aligned}
$$


We are now ready to prove Theorem 2 .

THEOREM 2. Let d be a positive nonsquare discriminant. Let $Q=(a, b, c)$ be a primitive, integral, binary quadratic form of discriminant $d$ with $a>0$. Let $\varepsilon=\frac{1}{2}\left(x_{0}+y_{0} \sqrt{d}\right)$ be as defined in (3). Set

$$
\alpha=\left(x_{0}-b y_{0}\right) / 2, \quad g=a y_{0} .
$$

Then $\alpha \in \mathbb{Z}$ and $(\alpha, g)=1$. Define $\alpha^{\prime} \in \mathbb{Z}$ by

$$
\alpha \alpha^{\prime} \equiv 1(\bmod g), \quad 0 \leq \alpha^{\prime}<g .
$$

For $l=1, \ldots,[(g-1) / 2]$ define $l^{*} \in \mathbb{Z}$ by

$$
l \alpha \equiv l^{*}(\bmod g), \quad 0 \leq l^{*}<g .
$$

For $l=1, \ldots,[(g-1) / 2]$ and $0 \leq t \leq 1$ set

$$
\begin{aligned}
F(\alpha, l, t, g)= & \frac{(t-\cos (2 \pi l \alpha / g)) \log \left(1-2 t^{\varepsilon} \cos (2 \pi l / g)+t^{2 \varepsilon}\right)}{t^{2}-2 t \cos (2 \pi l \alpha / g)+1} \\
& -\frac{2 \sin (2 \pi l \alpha / g) \tan ^{-1}\left(\frac{t^{\varepsilon} \sin (2 \pi l / g)}{1-t^{\varepsilon} \cos (2 \pi l / g)}\right)}{t^{2}-2 t \cos (2 \pi l \alpha / g)+1} .
\end{aligned}
$$

Then

$$
Z_{Q}(s)=\frac{\log \varepsilon}{\sqrt{d}} \cdot \frac{1}{s-1}+C_{Q}+O(s-1) \quad \text { as } s \rightarrow 1^{+},
$$

where

$$
\begin{gathered}
C_{Q}=V(d)+\frac{\pi^{2}}{6 a}+\frac{\log \varepsilon \log a}{\sqrt{d}}-\frac{1}{\sqrt{d}} W_{Q}, \\
V(d)=\frac{2 \gamma \log \varepsilon}{\sqrt{d}}+\frac{\log \varepsilon \log \left(\varepsilon y_{0}^{2}\right)}{\sqrt{d}}-\frac{1}{2 \sqrt{d}} \int_{0}^{\infty}\left(\frac{\log \left(u+\varepsilon^{2}\right)}{u+1}-\frac{\log (u+1)}{u+\varepsilon^{2}}\right) d u \\
+\frac{1}{\sqrt{d}} \int_{0}^{1}\left(\frac{1}{t \log t}-\frac{1}{t-1}\right) \log \left(\frac{1-t^{\varepsilon}}{1-t^{\varepsilon^{\prime}}}\right) d t,
\end{gathered}
$$

and

$$
\begin{aligned}
W_{Q} & =\int_{0}^{1} \sum_{l=1}^{[(g-1) / 2]}\left(F(\alpha, l, t, g)+F\left(\alpha^{\prime}, l, t, g\right)\right) d t \\
& -2 \sum_{l=1}^{[(g-1) / 2]}\left(\log \left(2 \sin \frac{\pi l}{g}\right) \log \left(2\left|\sin \frac{\pi l \alpha}{g}\right|\right)-\left(\frac{\pi}{2}-\frac{\pi l}{g}\right)\left(\frac{\pi}{2}-\frac{\pi l^{*}}{g}\right)\right) \\
& +\left(2 \int_{0}^{1} \frac{\log \left(1+t^{\varepsilon}\right)}{1+t} d t-\log ^{2} 2\right)\left(\frac{1+(-1)^{g}}{2}\right) .
\end{aligned}
$$


Proof. All the series and integrals appearing in Lemma 16 except the series for $\zeta(2 s-1)$ regarded as functions of the complex variable $s$ converge uniformly on compact subsets of the region $\operatorname{Re}(s)>1 / 2$, and so are analytic in this region. As $s \rightarrow 1^{+}$, we have

$$
\begin{aligned}
& \int_{\lambda_{1}}^{\infty} Q(t, 1)^{-s} d t \\
& \quad=\int_{\lambda_{1}}^{\infty} \frac{1}{Q(t, 1)} d t-\left(\int_{\lambda_{1}}^{\infty} \frac{\log Q(t, 1)}{Q(t, 1)} d t\right)(s-1)+O\left((s-1)^{2}\right) .
\end{aligned}
$$

We have $Q(t, 1)=a\left(t+t_{1}\right)\left(t+t_{2}\right)$, where

$$
t_{1}=\frac{b+\sqrt{d}}{2 a}, \quad t_{2}=\frac{b-\sqrt{d}}{2 a} .
$$

We note that by (38) and (40), $t+t_{1}$ and $t+t_{2}$ are positive for $t \geq \lambda_{1}$. Using these facts, it is easily shown that

$$
\int_{\lambda_{1}}^{\infty} \frac{1}{Q(t, 1)} d t=\frac{2 \log \varepsilon}{\sqrt{d}} .
$$

Also

$$
\begin{aligned}
\int_{\lambda_{1}}^{\infty} \frac{\log Q(t, 1)}{Q(t, 1)} d t & \\
= & \frac{1}{a\left(t_{1}-t_{2}\right)} \int_{\lambda_{1}}^{\infty} \log \left(a\left(t+t_{1}\right)\left(t+t_{2}\right)\right)\left(\frac{1}{t+t_{2}}-\frac{1}{t+t_{1}}\right) d t \\
= & \frac{1}{\sqrt{d}} \int_{\lambda_{1}}^{\infty} \log a\left(\frac{1}{t+t_{2}}-\frac{1}{t+t_{1}}\right) d t \\
& +\frac{1}{\sqrt{d}} \int_{\lambda_{1}}^{\infty} \log \left(\left(t+t_{1}\right)\left(t+t_{2}\right)\right)\left(\frac{1}{t+t_{2}}-\frac{1}{t+t_{1}}\right) d t \\
= & \frac{1}{\sqrt{d}} \int_{0}^{\infty} \log \left(\left(t+\lambda_{1}+t_{1}\right)\left(t+\lambda_{1}+t_{2}\right)\right)\left(\frac{1}{t+\lambda_{1}+t_{2}}-\frac{1}{t+\lambda_{1}+t_{1}}\right) d t \\
& +\frac{2 \log a \log \varepsilon}{\sqrt{d}} .
\end{aligned}
$$

Let

$$
\lambda_{0}=\frac{\sqrt{d}}{a\left(\varepsilon^{2}-1\right)},
$$


so that by (38), (40) and (46), we have

$$
\lambda_{1}+t_{1}=\varepsilon^{2} \lambda_{0}, \quad \lambda_{1}+t_{2}=\lambda_{0} .
$$

Hence

$$
\begin{aligned}
\int_{0}^{\infty} \log \left(\left(t+\lambda_{1}+\right.\right. & \left.\left.t_{1}\right)\left(t+\lambda_{1}+t_{2}\right)\right)\left(\frac{1}{t+\lambda_{1}+t_{2}}-\frac{1}{t+\lambda_{1}+t_{1}}\right) d t \\
= & \int_{0}^{\infty} \log \left(\left(t+\varepsilon^{2} \lambda_{0}\right)\left(t+\lambda_{0}\right)\right)\left(\frac{1}{t+\lambda_{0}}-\frac{1}{t+\varepsilon^{2} \lambda_{0}}\right) d t \\
= & \int_{0}^{\infty}\left(\frac{\log \left(t+\lambda_{0}\right)}{t+\lambda_{0}}-\frac{\log \left(t+\varepsilon^{2} \lambda_{0}\right)}{t+\varepsilon^{2} \lambda_{0}}\right) d t \\
& +\int_{0}^{\infty}\left(\frac{\log \left(t+\varepsilon^{2} \lambda_{0}\right)}{t+\lambda_{0}}-\frac{\log \left(t+\lambda_{0}\right)}{t+\varepsilon^{2} \lambda_{0}}\right) d t \\
= & 2 \log \varepsilon \log \left(\varepsilon \lambda_{0}\right)+\int_{0}^{\infty}\left(\frac{\log \left(t+\varepsilon^{2} \lambda_{0}\right)}{t+\lambda_{0}}-\frac{\log \left(t+\lambda_{0}\right)}{t+\varepsilon^{2} \lambda_{0}}\right) d t \\
= & 2 \log \varepsilon \log \left(\varepsilon \lambda_{0}\right)+2 \log \varepsilon \log \lambda_{0} \\
& +\int_{0}^{\infty}\left(\frac{\log \left(u+\varepsilon^{2}\right)}{u+1}-\frac{\log (u+1)}{u+\varepsilon^{2}}\right) d u,
\end{aligned}
$$

so that

$$
\begin{aligned}
& \int_{\lambda_{1}}^{\infty} \frac{\log Q(t, 1)}{Q(t, 1)} d t \\
& \quad=\frac{2 \log \varepsilon \log \left(a \varepsilon \lambda_{0}^{2}\right)}{\sqrt{d}}+\frac{1}{\sqrt{d}} \int_{0}^{\infty}\left(\frac{\log \left(u+\varepsilon^{2}\right)}{u+1}-\frac{\log (u+1)}{u+\varepsilon^{2}}\right) d u
\end{aligned}
$$

Using (45), (47) and (50), together with

$$
\begin{aligned}
a^{-s} \zeta(2 s) & =\frac{\pi^{2}}{6 a}+O(s-1), \\
\zeta(2 s-1) & =\frac{1 / 2}{s-1}+\gamma+O(s-1), \\
Q\left(\lambda_{1}, 1\right)^{-s} \sum_{y=1}^{\infty} \frac{P\left(\lambda_{1} y\right)}{y^{2 s}} & =Q\left(\lambda_{1}, 1\right)^{-1} \sum_{y=1}^{\infty} \frac{P\left(\lambda_{1} y\right)}{y^{2}}+O(s-1),
\end{aligned}
$$




$$
s \sum_{y=1}^{\infty} y^{-2 s} \int_{\lambda_{1}}^{\infty} \frac{(2 a t+b) P(t y)}{Q(t, 1)^{s+1}} d t=\sum_{y=1}^{\infty} y^{-2} \int_{\lambda_{1}}^{\infty} \frac{(2 a t+b) P(t y)}{Q(t, 1)^{2}} d t+O(s-1)
$$

in (44), where $\gamma$ denotes Euler's constant

$$
\gamma=\lim _{n \rightarrow \infty}\left(\sum_{i=1}^{n} \frac{1}{i}-\log n\right)=0.5772156649 \ldots,
$$

we obtain

$$
Z_{Q}(s)=\frac{\log \varepsilon}{\sqrt{d}} \cdot \frac{1}{s-1}+C_{Q}+O(s-1) \quad \text { as } s \rightarrow 1^{+},
$$

where

$$
\begin{aligned}
C_{Q}= & \frac{\pi^{2}}{6 a}+\frac{2 \gamma \log \varepsilon}{\sqrt{d}}-\frac{1}{2 \sqrt{d}} \int_{0}^{\infty}\left(\frac{\log \left(u+\varepsilon^{2}\right)}{u+1}-\frac{\log (u+1)}{u+\varepsilon^{2}}\right) d u \\
& -\frac{\log \varepsilon \log \left(a \varepsilon \lambda_{0}^{2}\right)}{\sqrt{d}}+Q\left(\lambda_{1}, 1\right)^{-1} \sum_{y=1}^{\infty} \frac{P\left(\lambda_{1} y\right)}{y^{2}} \\
& -\sum_{y=1}^{\infty} y^{-2} \int_{\lambda_{1}}^{\infty} \frac{(2 a t+b) P(t y)}{Q(t, 1)^{2}} d t
\end{aligned}
$$

Set

$$
K(d)=\frac{2 \gamma \log \varepsilon}{\sqrt{d}}-\frac{1}{2 \sqrt{d}} \int_{0}^{\infty}\left(\frac{\log \left(u+\varepsilon^{2}\right)}{u+1}-\frac{\log (u+1)}{u+\varepsilon^{2}}\right) d u,
$$

so that

$$
\begin{aligned}
C_{Q}= & K(d)+\frac{\pi^{2}}{6 a}-\frac{\log \varepsilon \log \left(a \varepsilon \lambda_{0}^{2}\right)}{\sqrt{d}}+\frac{1}{Q\left(\lambda_{1}, 1\right)} \sum_{y=1}^{\infty} \frac{P\left(\lambda_{1} y\right)}{y^{2}} \\
& -\sum_{y=1}^{\infty} y^{-2} \int_{\lambda_{1}}^{\infty} \frac{(2 a t+b) P(t y)}{Q(t, 1)^{2}} d t .
\end{aligned}
$$

We emphasize that $K(d)$ depends only on $d$ and not on the form $(a, b, c)$.

Throughout the rest of this section, we focus on transforming $C_{Q}$ into the form stated in Theorem 2. By (42) and (47), we have for $y>0$,

$$
\begin{aligned}
\sum_{x>\lambda_{1} y} Q\left(x y^{-1}, 1\right)^{-1} & =y \int_{\lambda_{1}}^{\infty} \frac{1}{Q(t, 1)} d t-\int_{\lambda_{1}}^{\infty} \frac{(2 a t+b) P(t y)}{Q(t, 1)^{2}} d t+\frac{P\left(\lambda_{1} y\right)}{Q\left(\lambda_{1}, 1\right)} \\
& =\frac{2 y \log \varepsilon}{\sqrt{d}}-\int_{\lambda_{1}}^{\infty} \frac{(2 a t+b) P(t y)}{Q(t, 1)^{2}} d t+\frac{P\left(\lambda_{1} y\right)}{Q\left(\lambda_{1}, 1\right)} .
\end{aligned}
$$


Hence

$$
\begin{aligned}
\sum_{y=1}^{\infty} y^{-2}\left(\sum_{x>\lambda_{1} y} Q\left(x y^{-1}, 1\right)^{-1}-\frac{2 y \log \varepsilon}{\sqrt{d}}\right) & \\
& =\sum_{y=1}^{\infty} y^{-2}\left(\frac{P\left(\lambda_{1} y\right)}{Q\left(\lambda_{1}, 1\right)}-\int_{\lambda_{1}}^{\infty} \frac{(2 a t+b) P(t y)}{Q(t, 1)^{2}} d t\right) .
\end{aligned}
$$

Using this in (54) gives

$$
\begin{aligned}
C_{Q}= & K(d)+\frac{\pi^{2}}{6 a}-\frac{\log \varepsilon \log \left(a \varepsilon \lambda_{0}^{2}\right)}{\sqrt{d}} \\
& +\sum_{y=1}^{\infty} y^{-2}\left(\sum_{x>\lambda_{1} y} Q\left(x y^{-1}, 1\right)^{-1}-\frac{2 y \log \varepsilon}{\sqrt{d}}\right) .
\end{aligned}
$$

By (46), we have, for $y>0$,

$$
\begin{aligned}
\sum_{x>\lambda_{1} y} Q & \left(x y^{-1}, 1\right)^{-1}=\sum_{x>\lambda_{1} y} \frac{1}{a\left(x y^{-1}+t_{1}\right)\left(x y^{-1}+t_{2}\right)} \\
& =\frac{y}{\sqrt{d}} \sum_{x=1+\left[\lambda_{1} y\right]}^{\infty}\left(\frac{1}{x+t_{2} y}-\frac{1}{x+t_{1} y}\right) \\
& =\frac{y}{\sqrt{d}} \sum_{m=0}^{\infty}\left(\frac{1}{m+1+\left[\lambda_{1} y\right]+t_{2} y}-\frac{1}{m+1+\left[\lambda_{1} y\right]+t_{1} y}\right) .
\end{aligned}
$$

Using (49), we note that $1+\left[\lambda_{1} y\right]+t_{2} y>\left(\lambda_{1}+t_{2}\right) y=\lambda_{0} y>0$ and $1+\left[\lambda_{1} y\right]+t_{1} y>\left(\lambda_{1}+t_{1}\right) y=\lambda_{0} \varepsilon^{2} y>0$. We recall the formula (see for example [10: formula 8.362, p. 952]), which is valid for $x>0$,

$$
-\psi(x)=\frac{1}{x}+\gamma+\sum_{m=1}^{\infty}\left(\frac{1}{x+m}-\frac{1}{m}\right),
$$

where $\psi(x)=\Gamma^{\prime}(x) / \Gamma(x)$. Hence, for $x_{1}>0, x_{2}>0$, we have

$$
\psi\left(x_{1}\right)-\psi\left(x_{2}\right)=\sum_{m=0}^{\infty}\left(\frac{1}{m+x_{2}}-\frac{1}{m+x_{1}}\right) .
$$

Using this in (56), we obtain, for $y>0$,

$$
\sum_{x>\lambda_{1} y} Q\left(x y^{-1}, 1\right)^{-1}=\frac{y}{\sqrt{d}}\left(\psi\left(1+\left[\lambda_{1} y\right]+t_{1} y\right)-\psi\left(1+\left[\lambda_{1} y\right]+t_{2} y\right)\right) .
$$

Hence, by (55), we have

$$
\begin{aligned}
C_{Q}= & K(d)+\frac{\pi^{2}}{6 a}-\frac{\log \varepsilon \log \left(a \varepsilon \lambda_{0}^{2}\right)}{\sqrt{d}} \\
& +\frac{1}{\sqrt{d}} \sum_{y=1}^{\infty} \frac{1}{y}\left(\psi\left(1+\left[\lambda_{1} y\right]+t_{1} y\right)-\psi\left(1+\left[\lambda_{1} y\right]+t_{2} y\right)-\log \varepsilon^{2}\right) .
\end{aligned}
$$


As in the proof of Lemma 13, we set

$$
\varepsilon^{\prime}=\left(x_{0}-y_{0} \sqrt{d}\right) / 2,
$$

so that

$$
\varepsilon \varepsilon^{\prime}=1
$$

as $x_{0}^{2}-d y_{0}^{2}=4$. Hence $\varepsilon^{\prime}\left(\varepsilon^{2}-1\right)=\varepsilon-\varepsilon^{\prime}=y_{0} \sqrt{d}$, so that by (48),

$$
\lambda_{0}=\frac{\sqrt{d}}{a\left(\varepsilon^{2}-1\right)}=\frac{\varepsilon^{\prime}}{a y_{0}},
$$

and by (38),

$$
\lambda=-b+\sqrt{d}+\frac{2 \sqrt{d}}{\varepsilon^{2}-1}=-b+\frac{\varepsilon-\varepsilon^{\prime}}{y_{0}}+\frac{2 \varepsilon^{\prime}}{y_{0}}=-b+\frac{\varepsilon+\varepsilon^{\prime}}{y_{0}} .
$$

Hence

$$
\lambda=-b+x_{0} / y_{0}
$$

and by (40),

$$
\lambda_{1}=\lambda /(2 a)=\alpha / g
$$

where

$$
g=g(a, d)=a y_{0}, \quad \alpha=\alpha(b, d)=\left(x_{0}-b y_{0}\right) / 2 .
$$

Since $b^{2}-4 a c=d$ and $x_{0}^{2}-d y_{0}^{2}=4$, we have

$$
x_{0}^{2}-b^{2} y_{0}^{2}=4-4 a c y_{0}^{2} \equiv 0(\bmod 4),
$$

so that $x_{0} \equiv b y_{0}(\bmod 2)$. Hence $\alpha$ is an integer. In fact

$$
(\alpha, g)=1
$$

since

$$
\alpha\left(\frac{x_{0}+b y_{0}}{2}\right)+g c y_{0}=1 .
$$

We have, by (40), (46), (62), (3) and (64),

$$
\lambda_{1}+t_{1}=\frac{\lambda+b+\sqrt{d}}{2 a}=\frac{x_{0} / y_{0}+\sqrt{d}}{2 a}=\frac{\varepsilon}{g},
$$

and similarly

$$
\lambda_{1}+t_{2}=\varepsilon^{\prime} / g
$$

For any positive integer $y$, we let

$$
r_{y}=g\left(\lambda_{1} y-\left[\lambda_{1} y\right]\right) \text {. }
$$

We note that $r_{y}$ is an integer since $r_{y}=\alpha y-g\left[\lambda_{1} y\right]$ by (63). Also

$$
0 \leq r_{y}<g \text {. }
$$


For $x_{1}>0$ and $x_{2}>0$ we have

$$
\log x_{1}-\log x_{2}=\int_{x_{2}}^{x_{1}} \frac{1}{t} d t=\int_{x_{2}}^{x_{1}} \int_{0}^{\infty} e^{-t u} d u d t
$$

so that

$$
\log x_{1}-\log x_{2}=\int_{0}^{\infty} \int_{x_{2}}^{x_{1}} e^{-t u} d t d u=\int_{0}^{\infty} \frac{e^{-x_{2} u}-e^{-x_{1} u}}{u} d u .
$$

On using the substitution $t=e^{-u}$, we obtain

$$
\log x_{1}-\log x_{2}=\int_{0}^{1} \frac{t^{x_{1}}-t^{x_{2}}}{t \log t} d t .
$$

Equation 3.311(6) in [10] gives

$$
\psi(x)+\gamma=\int_{0}^{\infty} \frac{e^{-u}-e^{-x u}}{1-e^{-u}} d u=\int_{0}^{1} \frac{1-t^{x-1}}{1-t} d t, \quad x>0 .
$$

Choosing $x=1+x_{1}>0$ and $x=1+x_{2}>0$, and subtracting, we obtain

$$
\psi\left(1+x_{1}\right)-\psi\left(1+x_{2}\right)=\int_{0}^{1} \frac{t^{x_{2}}-t^{x_{1}}}{1-t} d t .
$$

Appealing to (60) and (66)-(71), we obtain

$$
\begin{aligned}
& \sum_{y=1}^{\infty} \frac{1}{y}\left(\psi\left(1+\left[\lambda_{1} y\right]+t_{1} y\right)-\psi\left(1+\left[\lambda_{1} y\right]+t_{2} y\right)-\log \varepsilon^{2}\right) \\
&=\sum_{y=1}^{\infty} \frac{1}{y}\left(\psi\left(1+\left(\lambda_{1}+t_{1}\right) y-\left(\lambda_{1} y-\left[\lambda_{1} y\right]\right)\right)\right. \\
&\left.-\psi\left(1+\left(\lambda_{1}+t_{2}\right) y-\left(\lambda_{1} y-\left[\lambda_{1} y\right]\right)\right)-\log \varepsilon^{2}\right) \\
&= \sum_{y=1}^{\infty} \frac{1}{y}\left(\psi\left(1+\frac{\varepsilon y}{g}-\frac{r_{y}}{g}\right)-\psi\left(1+\frac{\varepsilon^{\prime} y}{g}-\frac{r_{y}}{g}\right)-\left(\log (\varepsilon y)-\log \left(\varepsilon^{\prime} y\right)\right)\right) \\
&= \sum_{y=1}^{\infty} \frac{1}{y}\left(\int_{0}^{1} \frac{t^{\left(\varepsilon^{\prime} y-r_{y}\right) / g}-t^{\left(\varepsilon y-r_{y}\right) / g}}{1-t} d t-\int_{0}^{1} \frac{t^{\varepsilon y}-t^{\varepsilon^{\prime} y}}{t \log t} d t\right) \\
&= \sum_{y=1}^{\infty} \frac{1}{y}\left(g \int_{0}^{1} \frac{t^{\varepsilon^{\prime} y}-t^{\varepsilon y}}{1-t^{g}} t^{g-1-r_{y}} d t-\int_{0}^{1} \frac{t^{\varepsilon y}-t^{\varepsilon^{\prime} y}}{t \log t} d t\right) \\
&= \int_{0}^{1} \sum_{y=1}^{\infty} \frac{t^{\varepsilon y}-t^{\varepsilon^{\prime} y}}{t y}\left(-\frac{1}{\log t}+\frac{g t^{g-r_{y}}}{t^{g}-1}\right) d t,
\end{aligned}
$$


so that

$$
\begin{aligned}
\sum_{y=1}^{\infty} \frac{1}{y}(\psi(1+ & {\left.\left.\left[\lambda_{1} y\right]+t_{1} y\right)-\psi\left(1+\left[\lambda_{1} y\right]+t_{2} y\right)-\log \varepsilon^{2}\right) } \\
& =\int_{0}^{1}\left(\frac{1}{t \log t} \log \left(\frac{1-t^{\varepsilon}}{1-t^{\varepsilon^{\prime}}}\right)+g \sum_{y=1}^{\infty} \frac{t^{\varepsilon y}-t^{\varepsilon^{\prime} y}}{y\left(t^{g}-1\right)} t^{g-r_{y}-1}\right) d t
\end{aligned}
$$

Let $0<t<1$. We have

$$
\begin{aligned}
\sum_{y=1}^{\infty} \frac{g}{y} \cdot \frac{t^{\varepsilon y}-t^{\varepsilon^{\prime} y}}{t^{g}-1} t^{g-r_{y}-1} & =\sum_{k=0}^{g-1} \sum_{r_{y}=k} \frac{t^{\varepsilon y}-t^{\varepsilon^{\prime} y}}{y} \cdot \frac{g t^{g-1-k}}{t^{g}-1} \\
& =\sum_{k=0}^{g-1} \sum_{\alpha y \equiv k(\bmod g)} \frac{t^{\varepsilon y}-t^{\varepsilon^{\prime} y}}{y} \cdot \frac{g t^{g-k-1}}{t^{g}-1} .
\end{aligned}
$$

Let

$$
\theta=e^{2 \pi i / g}
$$

Then

$$
\frac{g t^{g-k-1}}{t^{g}-1}=\frac{g t^{g-k-1}}{(t-\theta) \cdots\left(t-\theta^{g}\right)}=\sum_{l=1}^{g} \frac{A_{l, k}}{t-\theta^{l}},
$$

where $A_{l, k}=g \theta^{-l(k+1)} / \prod_{j \neq l}\left(\theta^{l}-\theta^{j}\right)$. But, for $1 \leq l \leq g$, we have

$$
\prod_{j \neq l}\left(\theta^{l}-\theta^{j}\right)=\theta^{l(g-1)} \prod_{j \neq l}\left(1-\theta^{j-l}\right)=\theta^{-l} \prod_{i=1}^{g-1}\left(1-\theta^{i}\right)=g \theta^{-l},
$$

so that

$$
A_{l, k}=\theta^{-l k}
$$

Thus, by (73), (75) and (76), we have

$$
\begin{aligned}
\sum_{y=1}^{\infty} \frac{g}{y} \cdot \frac{t^{\varepsilon y}-t^{\varepsilon^{\prime} y}}{t^{g}-1} t^{g-r_{y}-1} & =\sum_{k=0}^{g-1} \sum_{\alpha y \equiv k(\bmod g)} \frac{t^{\varepsilon y}-t^{\varepsilon^{\prime} y}}{y} \sum_{l=1}^{g} \frac{\theta^{-l k}}{t-\theta^{l}} \\
& =\sum_{l=1}^{g} \frac{1}{t-\theta^{l}} \sum_{k=0}^{g-1} \sum_{\alpha y \equiv k(\bmod g)} \frac{t^{\varepsilon y}-t^{\varepsilon^{\prime} y}}{y} \theta^{-l k} \\
& =\sum_{l=1}^{g} \frac{1}{t-\theta^{l}} \sum_{k=0}^{g-1} \sum_{\alpha y \equiv k(\bmod g)} \frac{t^{\varepsilon y}-t^{\varepsilon^{\prime} y}}{y} \theta^{-l \alpha y} \\
& =\sum_{l=1}^{g} \frac{1}{t-\theta^{l}} \sum_{y=1}^{\infty} \frac{t^{\varepsilon y}-t^{\varepsilon^{\prime} y}}{y} \theta^{-l \alpha y}
\end{aligned}
$$




$$
=-\sum_{l=1}^{g} \frac{1}{t-\theta^{l}}\left(\log \left(1-\theta^{-l \alpha} t^{\varepsilon}\right)-\log \left(1-\theta^{-l \alpha} t^{\varepsilon^{\prime}}\right)\right),
$$

where the principal values of the logarithms are taken. Using this in (72) gives

$$
\begin{aligned}
\sum_{y=1}^{\infty} \frac{1}{y}(\psi(1+ & {\left.\left.\left[\lambda_{1} y\right]+t_{1} y\right)-\psi\left(1+\left[\lambda_{1} y\right]+t_{2} y\right)-\log \varepsilon^{2}\right) } \\
= & \int_{0}^{1}\left(\frac{1}{t \log t} \log \left(\frac{1-t^{\varepsilon}}{1-t^{\varepsilon^{\prime}}}\right)\right. \\
& \left.-\sum_{l=1}^{g} \frac{1}{t-\theta^{l}}\left(\log \left(1-\theta^{-l \alpha} t^{\varepsilon}\right)-\log \left(1-\theta^{-l \alpha} t^{\varepsilon^{\prime}}\right)\right)\right) d t \\
= & \int_{0}^{1}\left(\frac{1}{t \log t}-\frac{1}{t-1}\right) \log \left(\frac{1-t^{\varepsilon}}{1-t^{\varepsilon^{\prime}}}\right) d t \\
& -\int_{0}^{1} \sum_{l=1}^{g-1} \frac{1}{t-\theta^{l}}\left(\log \left(1-\theta^{-l \alpha} t^{\varepsilon}\right)-\log \left(1-\theta^{-l \alpha} t^{\varepsilon^{\prime}}\right)\right) d t .
\end{aligned}
$$

For $1 \leq l \leq g-1$, we have $\theta^{l} \neq 1, \theta^{-l \alpha} \neq 1$ (by (65)), and

$$
\begin{aligned}
& \int_{0}^{1} \frac{1}{t-\theta^{l}} \log \left(1-\theta^{-l \alpha} t^{\varepsilon^{\prime}}\right) d t=-\theta^{-l} \int_{0}^{1} \frac{1}{1-\theta^{-l} t} \log \left(1-\theta^{-l \alpha} t^{\varepsilon^{\prime}}\right) d t \\
& =\left[\log \left(1-\theta^{-l \alpha} t^{\varepsilon^{\prime}}\right) \log \left(1-\theta^{-l} t\right)\right]_{0}^{1}-\int_{0}^{1} \frac{\log \left(1-\theta^{-l} t\right)}{1-\theta^{-l \alpha} t^{\varepsilon^{\prime}}}\left(-\theta^{-l \alpha}\right) \varepsilon^{\prime} t^{\varepsilon^{\prime}-1} d t \\
& =\log \left(1-\theta^{-l \alpha}\right) \log \left(1-\theta^{-l}\right)-\int_{0}^{1} \frac{\log \left(1-\theta^{-l} t^{\varepsilon}\right)}{t-\theta^{l \alpha}} d t .
\end{aligned}
$$

Hence, by (78), (77), (58), (61), (60) and (53), we obtain

$$
C_{Q}=V(d)+\frac{\pi^{2}}{6 a}+\frac{\log \varepsilon \log a}{\sqrt{d}}-\frac{1}{\sqrt{d}} W_{Q},
$$

where $V(d)$ is defined in the statement of Theorem 2 and

$$
\begin{aligned}
W_{Q}= & \sum_{l=1}^{g-1}\left(\int_{0}^{1} \frac{\log \left(1-\theta^{-l \alpha} t^{\varepsilon}\right)}{t-\theta^{l}} d t+\int_{0}^{1} \frac{\log \left(1-\theta^{-l} t^{\varepsilon}\right)}{t-\theta^{l \alpha}} d t\right) \\
& -\sum_{l=1}^{g-1} \log \left(1-\theta^{-l \alpha}\right) \log \left(1-\theta^{-l}\right) .
\end{aligned}
$$


We emphasize that $V(d)$ depends only on $d$ and not on the form $(a, b, c)$.

Since $(\alpha, g)=1$, we may choose an integer $\alpha^{\prime}$ such that

$$
\alpha \alpha^{\prime} \equiv 1(\bmod g) \text {. }
$$

Changing the variable from $l$ to $l \alpha^{\prime}$ in the first sum in (80), we obtain

$$
\begin{aligned}
W_{Q}= & \sum_{l=1}^{g-1} \int_{0}^{1} \log \left(1-\theta^{-l} t^{\varepsilon}\right)\left(\frac{1}{t-\theta^{l \alpha^{\prime}}}+\frac{1}{t-\theta^{l \alpha}}\right) d t \\
& -\sum_{l=1}^{g-1} \log \left(1-\theta^{-l \alpha}\right) \log \left(1-\theta^{-l}\right) \\
= & S_{1}(\alpha)+S_{1}\left(\alpha^{\prime}\right)-\sum_{l=1}^{g-1} \log \left(1-\theta^{-l \alpha}\right) \log \left(1-\theta^{-l}\right),
\end{aligned}
$$

where

$$
S_{1}(\alpha)=\sum_{l=1}^{g-1} \int_{0}^{1} \frac{\log \left(1-\theta^{-l} t^{\varepsilon}\right)}{t-\theta^{l \alpha}} d t .
$$

We set

$$
F(\alpha, l, t, g)=\frac{\log \left(1-\theta^{-l} t^{\varepsilon}\right)}{t-\theta^{l \alpha}}+\frac{\log \left(1-\theta^{l} t^{\varepsilon}\right)}{t-\theta^{-l \alpha}} .
$$

We first consider the case when $g$ is odd. Let $g=2 m+1$ where $m \geq 1$. We note that $W_{Q}=0$ if $g=1$. Then

$$
\begin{aligned}
S_{1}(\alpha) & =\sum_{l=1}^{2 m} \int_{0}^{1} \frac{\log \left(1-\theta^{-l} t^{\varepsilon}\right)}{t-\theta^{l \alpha}} d t \\
& =\int_{0}^{1}\left(\sum_{l=1}^{m} \frac{\log \left(1-\theta^{-l} t^{\varepsilon}\right)}{t-\theta^{l \alpha}}+\sum_{l=m+1}^{2 m} \frac{\log \left(1-\theta^{2 m+1-l} t^{\varepsilon}\right)}{t-\theta^{-(2 m+1-l) \alpha}}\right) d t \\
& =\int_{0}^{1} \sum_{l=1}^{m}\left(\frac{\log \left(1-\theta^{-l} t^{\varepsilon}\right)}{t-\theta^{l \alpha}}+\frac{\log \left(1-\theta^{l} t^{\varepsilon}\right)}{t-\theta^{-l \alpha}}\right) d t \\
& =\int_{0}^{1} \sum_{l=1}^{m} F(\alpha, l, t, g) d t .
\end{aligned}
$$

Similarly we have

$$
\begin{aligned}
\sum_{l=1}^{g-1} \log (1 & \left.-\theta^{-l \alpha}\right) \log \left(1-\theta^{-l}\right) \\
& =\sum_{l=1}^{m}\left(\log \left(1-\theta^{-l \alpha}\right) \log \left(1-\theta^{-l}\right)+\log \left(1-\theta^{l \alpha}\right) \log \left(1-\theta^{l}\right)\right) .
\end{aligned}
$$


Hence, by (82), we have

$$
\begin{aligned}
W_{Q}= & \int_{0}^{1} \sum_{l=1}^{[(g-1) / 2]}\left(F(\alpha, l, t, g)+F\left(\alpha^{\prime}, l, t, g\right)\right) d t \\
& -\sum_{l=1}^{[(g-1) / 2]}\left(\log \left(1-\theta^{-l \alpha}\right) \log \left(1-\theta^{-l}\right)+\log \left(1-\theta^{l \alpha}\right) \log \left(1-\theta^{l}\right)\right) .
\end{aligned}
$$

Similarly, for $g$ even, we obtain

$$
\begin{aligned}
W_{Q}= & \int_{0}^{1} \sum_{l=1}^{[(g-1) / 2]}\left(F(\alpha, l, t, g)+F\left(\alpha^{\prime}, l, t, g\right)\right) d t+2 \int_{0}^{1} \frac{\log \left(1+t^{\varepsilon}\right)}{1+t} d t \\
& -\sum_{l=1}^{[(g-1) / 2]}\left(\log \left(1-\theta^{-l \alpha}\right) \log \left(1-\theta^{-l}\right)+\log \left(1-\theta^{l \alpha}\right) \log \left(1-\theta^{l}\right)\right)-\log ^{2} 2 .
\end{aligned}
$$

Thus, for all $g$, we have

$$
\begin{aligned}
W_{Q}= & \int_{0}^{1} \sum_{l=1}^{[(g-1) / 2]}\left(F(\alpha, l, t, g)+F\left(\alpha^{\prime}, l, t, g\right)\right) d t \\
& -\sum_{l=1}^{[(g-1) / 2]}\left(\log \left(1-\theta^{-l \alpha}\right) \log \left(1-\theta^{-l}\right)+\log \left(1-\theta^{l \alpha}\right) \log \left(1-\theta^{l}\right)\right) \\
& +\left(2 \int_{0}^{1} \frac{\log \left(1+t^{\varepsilon}\right)}{1+t} d t-\log ^{2} 2\right) \frac{1+(-1)^{g}}{2} .
\end{aligned}
$$

Explicitly calculating the logarithms occurring in (84), we obtain, after some simplification,

$$
\begin{aligned}
F(\alpha, l, t, g)= & \frac{(t-\cos (2 \pi l \alpha / g)) \log \left(1-2 t^{\varepsilon} \cos (2 \pi l / g)+t^{2 \varepsilon}\right)}{t^{2}-2 t \cos (2 \pi l \alpha / g)+1} \\
& -\frac{2 \sin (2 \pi l \alpha / g) \tan ^{-1}\left(\frac{t^{\varepsilon} \sin (2 \pi l / g)}{1-t^{\varepsilon} \cos (2 \pi l / g)}\right)}{t^{2}-2 t \cos (2 \pi l \alpha / g)+1},
\end{aligned}
$$

for $1 \leq l \leq[(g-1) / 2], 0 \leq t \leq 1$. Similarly, for $1 \leq l \leq[(g-1) / 2]$, we obtain

$$
\begin{aligned}
\log (1 & \left.-\theta^{-l \alpha}\right) \log \left(1-\theta^{-l}\right)+\log \left(1-\theta^{l \alpha}\right) \log \left(1-\theta^{l}\right) \\
& =2\left(\log \left(2 \sin \frac{\pi l}{g}\right) \log \left(2\left|\sin \frac{\pi l \alpha}{g}\right|\right)-\left(\frac{\pi}{2}-\frac{\pi l}{g}\right) \tan ^{-1}\left(\cot \frac{\pi l \alpha}{g}\right)\right) .
\end{aligned}
$$

Let

$$
l \alpha \equiv l^{*}(\bmod g)
$$


where $0 \leq l^{*}<g$. Then

$$
\tan ^{-1}\left(\cot \frac{\pi l \alpha}{g}\right)=\frac{\pi}{2}-\frac{\pi l^{*}}{g} .
$$

Thus our final formula for $W_{Q}$ is

$$
\begin{aligned}
W_{Q}= & \int_{0}^{1} \sum_{l=1}^{[(g-1) / 2]}\left(F(\alpha, l, t, g)+F\left(\alpha^{\prime}, l, t, g\right)\right) d t \\
& -2 \sum_{l=1}^{[(g-1) / 2]}\left(\log \left(2 \sin \frac{\pi l}{g}\right) \log \left(2\left|\sin \frac{\pi l \alpha}{g}\right|\right)\right. \\
& \left.-\left(\frac{\pi}{2}-\frac{\pi l}{g}\right)\left(\frac{\pi}{2}-\frac{\pi l^{*}}{g}\right)\right) \\
& +\left(2 \int_{0}^{1} \frac{\log \left(1+t^{\varepsilon}\right)}{1+t} d t-\log ^{2} 2\right)\left(\frac{1+(-1)^{g}}{2}\right) .
\end{aligned}
$$

This completes our proof of Theorem 2.

4. Behaviour of $\sum_{n=1}^{\infty} R_{G}(n, d) / n^{s}$ near $s=1$. Proofs of Theorems 3 and 4. Let $K \in H(d)$, where $d$ is a positive nonsquare discriminant, and let $Q=(a, b, c) \in K$ with $a>0$. For $s>1$ we have

$$
Z_{Q}(s)=\sum_{n=1}^{\infty} \frac{R_{Q}(n, d)}{n^{s}}=\sum_{n=1}^{\infty} \frac{R_{K}(n, d)}{n^{s}} .
$$

Thus, for $G \in G(d)$, we see that

$$
\sum_{n=1}^{\infty} \frac{R_{G}(n, d)}{n^{s}}=\sum_{K \in G} \sum_{n=1}^{\infty} \frac{R_{K}(n, d)}{n^{s}}
$$

converges for $s>1$. We now evaluate the Dirichlet series on the left hand side of (90) explicitly using the formula for $R_{G}(n, d)$ given in Theorem 1. We prove

Theorem 3. Let $G \in G(d)$. For $s>1$, we have

$$
\begin{aligned}
\sum_{n=1}^{\infty} & \frac{R_{G}(n, d)}{n^{s}} \\
= & \frac{h(d) \log \varepsilon(d)}{2^{t(d)+1}} \sum_{m \mid f} \frac{1}{\log \varepsilon\left(d / m^{2}\right) h\left(d / m^{2}\right)} \cdot \frac{1}{m^{2 s}} \sum_{d_{1} \in F\left(d / m^{2}\right)} \gamma_{d_{1}}(G) \\
& \times \prod_{p \mid f / m}\left(1-\left(\frac{d_{1}}{p}\right) p^{-s}\right)\left(1-\left(\frac{\Delta\left(d / d_{1}\right)}{p}\right) p^{-s}\right) L\left(s, d_{1}\right) L\left(s, \Delta\left(d / d_{1}\right)\right),
\end{aligned}
$$


where the Dirichlet L-series $L(s, d)$ is defined for $s>0$ by

$$
L(s, d)=\sum_{n=1}^{\infty} \frac{\left(\frac{d}{n}\right)}{n^{s}} .
$$

Proof. By Theorem 1, we have

$$
\begin{aligned}
\sum_{n=1}^{\infty} & \frac{R_{G}(n, d)}{n^{s}} \\
= & \sum_{\substack{n=1 \\
\text { Null }(n, d)=\emptyset}}^{\infty} \frac{1}{n^{s}} \cdot \frac{\log \varepsilon(d)}{\log \varepsilon\left(d / M(n, d)^{2}\right)} \cdot \frac{h(d)}{h\left(d / M(n, d)^{2}\right)} \cdot \frac{1}{2^{t(d)+1}} \\
& \times \sum_{\substack{d_{1} \in F\left(d / M(n, d)^{2}\right) \\
=}} \frac{\gamma_{d_{1}}(G) S\left(n / M(n, d)^{2}, d_{1}, d / M(n, d)^{2} d_{1}\right)}{2^{t(d)+1}} \sum_{m \mid f} \frac{1}{\log \varepsilon\left(d / m^{2}\right) h\left(d / m^{2}\right)} \sum_{d_{1} \in F\left(d / m^{2}\right)} \gamma_{d_{1}}(G) \\
& \times \sum_{\substack{n=1 \\
\text { Null }(n, d)=\emptyset \\
M(n, d)=m}}^{\infty} \frac{S\left(n / m^{2}, d_{1}, d / m^{2} d_{1}\right)}{n^{s}} .
\end{aligned}
$$

For $m^{2} \mid n$ and $m \mid f$ it is easy to check that

$$
\begin{aligned}
\operatorname{Null}(n, d)=\emptyset & \Leftrightarrow \operatorname{Null}\left(n / m^{2}, d / m^{2}\right)=\emptyset, \\
M(n, d)=m & \Leftrightarrow M\left(n / m^{2}, d / m^{2}\right)=1 .
\end{aligned}
$$

Hence for $m \mid f$ we have

$$
\begin{gathered}
\sum_{\substack{n=1 \\
\text { Null }(n, d)=\emptyset \\
M(n, d)=m}}^{\infty} \frac{S\left(n / m^{2}, d_{1}, d / m^{2} d_{1}\right)}{n^{s}}=\sum_{\substack{n=1 \\
m^{2} \mid n \\
\text { Null }(n, d)=\emptyset \\
M(n, d)=m}}^{\infty} \frac{S\left(n / m^{2}, d_{1}, d / m^{2} d_{1}\right)}{n^{s}} \\
=\sum_{\substack{n=1 \\
m^{2} \mid n \\
\operatorname{Null}\left(n / m^{2}, d / m^{2}\right)=\emptyset \\
M\left(n / m^{2}, d / m^{2}\right)=1}}^{\infty} \frac{S\left(n / m^{2}, d_{1}, d / m^{2} d_{1}\right)}{n^{s}} \\
=\sum_{\substack{n=1 \\
m^{2} \mid n \\
\left(n / m^{2}, f / m\right)=1}}^{\infty} \frac{S\left(n / m^{2}, d_{1}, d / m^{2} d_{1}\right)}{n^{s}} \quad \text { (by Lemma 6) }
\end{gathered}
$$




$$
\begin{aligned}
& =\sum_{\substack{N=1 \\
(N, f / m)=1}}^{\infty} \frac{S\left(N, d_{1}, d / m^{2} d_{1}\right)}{\left(m^{2} N\right)^{s}} \\
& =m^{-2 s} \sum_{\substack{N=1 \\
(N, f / m)=1}}^{\infty} \frac{1}{N^{s}} \sum_{\mu \nu=N}\left(\frac{d_{1}}{\mu}\right)\left(\frac{d / m^{2} d_{1}}{\nu}\right) \\
& =m^{-2 s} \sum_{(\mu, f / m)=1} \frac{1}{\mu^{s}}\left(\frac{d_{1}}{\mu}\right) \sum_{(\nu, f / m)=1} \frac{1}{\nu^{s}}\left(\frac{d / m^{2} d_{1}}{\nu}\right) .
\end{aligned}
$$

As $d_{1} \in F\left(d / m^{2}\right)$, by Lemma $1(\mathrm{~d})$ we have

$$
f\left(d_{2}\right) \mid f / m, \quad \text { where } \quad d_{2}=\frac{d / m^{2}}{d_{1}} .
$$

Thus for $(\nu, f / m)=1$ we have $\left(\nu, f\left(d_{2}\right)\right)=1$ so that

$$
\begin{aligned}
\left(\frac{d / m^{2} d_{1}}{\nu}\right) & =\left(\frac{d_{2}}{\nu}\right)=\left(\frac{\Delta\left(d_{2}\right) f\left(d_{2}\right)^{2}}{\nu}\right)=\left(\frac{\Delta\left(d_{2}\right)}{\nu}\right) \\
& =\left(\frac{\Delta\left(d_{2} m^{2}\right)}{\nu}\right)=\left(\frac{\Delta\left(d / d_{1}\right)}{\nu}\right) .
\end{aligned}
$$

Hence

$$
\begin{aligned}
& \sum_{\substack{n=1 \\
\text { Null }(n, d)=\emptyset \\
M(n, d)=m}}^{\infty} \frac{S\left(n / m^{2}, d_{1}, d / m^{2} d_{1}\right)}{n^{s}} \\
& =m^{-2 s} \sum_{(\mu, f / m)=1} \frac{1}{\mu^{s}}\left(\frac{d_{1}}{\mu}\right) \sum_{(\nu, f / m)=1} \frac{1}{\nu^{s}}\left(\frac{\Delta\left(d / d_{1}\right)}{\nu}\right) \\
& =m^{-2 s} L\left(s, d_{1}\right) \prod_{p \mid f / m}\left(1-\left(\frac{d_{1}}{p}\right) p^{-s}\right) \\
& \quad \times L\left(s, \Delta\left(d / d_{1}\right)\right) \prod_{p \mid f / m}\left(1-\left(\frac{\Delta\left(d / d_{1}\right)}{p}\right) p^{-s}\right) .
\end{aligned}
$$

The required result now follows on using (91).

We next determine the behaviour of $\sum_{n=1}^{\infty} R_{G}(n, d) / n^{s}$ as $s \rightarrow 1^{+}$.

Theorem 4. Let $G \in G(d), d>0$. As $s \rightarrow 1^{+}$we have

$$
\sum_{n=1}^{\infty} \frac{R_{G}(n, d)}{n^{s}}=\frac{h(d) \log \varepsilon(d)}{2^{t(d)} \sqrt{d}} \cdot \frac{1}{s-1}+B(d)+\beta(d, G)+O(s-1)
$$


where $B(d)$ depends only on $d$ and not on $G$ (see (100)) and

$$
\begin{aligned}
\beta(d, G)= & \frac{1}{2^{t(d)+1}} \sum_{\substack{d_{1} \in F(d) \\
d_{1} \notin\{1, \Delta\}}} \gamma_{d_{1}}(G) L\left(1, d_{1}\right) L\left(1, \Delta\left(d / d_{1}\right)\right) \\
& \times \sum_{m \mid f\left(d / d_{1}\right)} \frac{1}{m} \prod_{\substack{p \mid m \\
p \nmid f / m}}\left(1-\left(\frac{\Delta}{p}\right) p^{-1}\right) \prod_{p \mid f / m}\left(1-\left(\frac{d_{1}}{p}\right) p^{-1}\right) \\
& \times \prod_{p \mid f / m}\left(1-\left(\frac{\Delta\left(d / d_{1}\right)}{p}\right) p^{-1}\right) .
\end{aligned}
$$

Proof. By Theorem 3, we have

$$
\sum_{n=1}^{\infty} \frac{R_{G}(n, d)}{n^{s}}=S_{1}+S_{2},
$$

where

$$
\begin{aligned}
S_{1}= & \frac{h(d) \log \varepsilon(d)}{2^{t(d)+1}} \sum_{m \mid f} \frac{1}{\log \varepsilon\left(d / m^{2}\right) h\left(d / m^{2}\right)} \cdot \frac{1}{m^{2 s}} \\
& \times 2 \zeta(s) L(s, \Delta) \prod_{p \mid f / m}\left(1-p^{-s}\right)\left(1-\left(\frac{\Delta}{p}\right) p^{-s}\right),
\end{aligned}
$$

and

$$
\begin{aligned}
S_{2}= & \frac{h(d) \log \varepsilon(d)}{2^{t(d)+1}} \sum_{m \mid f} \frac{1}{\log \varepsilon\left(d / m^{2}\right) h\left(d / m^{2}\right)} \cdot \frac{1}{m^{2 s}} \sum_{\substack{d_{1} \in F\left(d / m^{2}\right) \\
d_{1} \notin\{1, \Delta\}}} \gamma_{d_{1}}(G) \\
& \times \prod_{p \mid f / m}\left(1-\left(\frac{d_{1}}{p}\right) p^{-s}\right)\left(1-\left(\frac{\Delta\left(d / d_{1}\right)}{p}\right) p^{-s}\right) \\
& \times L\left(s, d_{1}\right) L\left(s, \Delta\left(d / d_{1}\right)\right) .
\end{aligned}
$$

We first deal with $S_{2}$. We have

$$
\begin{aligned}
S_{2}= & \frac{1}{2^{t(d)+1}} \sum_{m \mid f} \frac{h(d) \log \varepsilon(d)}{h\left(d / m^{2}\right) \log \varepsilon\left(d / m^{2}\right)} \cdot \frac{1}{m^{2}} \sum_{\substack{d_{1} \in F\left(d / m^{2}\right) \\
d_{1} \notin\{1, \Delta\}}} \gamma_{d_{1}}(G) \\
& \times \prod_{p \mid f / m}\left(1-\left(\frac{d_{1}}{p}\right) p^{-1}\right)\left(1-\left(\frac{\Delta\left(d / d_{1}\right)}{p}\right) p^{-1}\right) \\
& \times L\left(1, d_{1}\right) L\left(1, \Delta\left(d / d_{1}\right)\right)+O(s-1) .
\end{aligned}
$$

We recall (see for example [12: Theorem 11.2, p. 322]), that if $l$ is a nonsquare 
discriminant and $d=l m^{2}$ then

$$
\frac{L(1, d)}{L(1, l)}=\prod_{p \mid m}\left(1-\left(\frac{l}{p}\right) p^{-1}\right) .
$$

By (95) and Dirichlet's class number formula (see for example [12: Theorem 10.1, p. 321]), we have

$$
\begin{aligned}
\frac{h(d) \log \varepsilon(d)}{h\left(d / m^{2}\right) \log \varepsilon\left(d / m^{2}\right)} & =\frac{\sqrt{d} L(1, d)}{\sqrt{d / m^{2}} L\left(1, d / m^{2}\right)} \\
& =m \prod_{p \mid m}\left(1-\left(\frac{d / m^{2}}{p}\right) p^{-1}\right) \\
& =m \prod_{\substack{p \mid m \\
p \nmid f / m}}\left(1-\left(\frac{\Delta}{p}\right) p^{-1}\right) .
\end{aligned}
$$

Using (96), we obtain

$$
\begin{aligned}
S_{2}= & \frac{1}{2^{t(d)+1}} \sum_{m \mid f} \frac{1}{m} \prod_{\substack{p \mid m \\
p \nmid f / m}}\left(1-\left(\frac{\Delta}{p}\right) p^{-1}\right) \sum_{\substack{d_{1} \in F\left(d / m^{2}\right) \\
d_{1} \notin\{1, \Delta\}}} \gamma_{d_{1}}(G) \\
& \times \prod_{p \mid f / m}\left(1-\left(\frac{d_{1}}{p}\right) p^{-1}\right)\left(1-\left(\frac{\Delta\left(d / d_{1}\right)}{p}\right) p^{-1}\right) \\
& \times L\left(1, d_{1}\right) L\left(1, \Delta\left(d / d_{1}\right)\right)+O(s-1) .
\end{aligned}
$$

Interchanging the orders of summation and appealing to Lemma 1(e), we obtain

$$
\begin{aligned}
S_{2}= & \frac{1}{2^{t(d)+1}} \sum_{\substack{d_{1} \in F(d) \\
d_{1} \notin\{1, \Delta\}}} \gamma_{d_{1}}(G) L\left(1, d_{1}\right) L\left(1, \Delta\left(d / d_{1}\right)\right) \sum_{m \mid f\left(d / d_{1}\right)} \frac{1}{m} \\
& \times \prod_{\substack{p \mid m \\
p \nmid f / m}}\left(1-\left(\frac{\Delta}{p}\right) p^{-1}\right) \prod_{p \mid f / m}\left(1-\left(\frac{d_{1}}{p}\right) p^{-1}\right) \\
& \times \prod_{p \mid f / m}\left(1-\left(\frac{\Delta\left(d / d_{1}\right)}{p}\right) p^{-1}\right)+O(s-1) \\
= & \beta(d, G)+O(s-1) .
\end{aligned}
$$

By (93) and (96), we have

$$
S_{1}=\frac{\zeta(s)}{2^{t(d)}} A(s, d)
$$


where

$$
\begin{aligned}
A(s, d)= & L(s, \Delta) \sum_{m \mid f} \frac{1}{m^{2 s-1}} \prod_{\substack{p \mid m \\
p \nmid f / m}}\left(1-\left(\frac{\Delta}{p}\right) p^{-1}\right) \\
& \times \prod_{p \mid f / m}\left(1-p^{-s}\right)\left(1-\left(\frac{\Delta}{p}\right) p^{-s}\right) .
\end{aligned}
$$

Hence, we obtain

$$
S_{1}=\frac{A(1, d)}{2^{t(d)}} \cdot \frac{1}{s-1}+B(d)+O(s-1),
$$

where

$$
B(d)=\left(A^{\prime}(1, d)+\gamma A(1, d)\right) / 2^{t(d)} .
$$

We emphasize that $B(d)$ depends only on $d$ and not on the genus $G$. By (95) and Dirichlet's class number formula, we obtain

$$
\begin{aligned}
L(1, \Delta) & =L(1, d) \prod_{p \mid f}\left(1-\left(\frac{\Delta}{p}\right) p^{-1}\right)^{-1} \\
& =\frac{h(d) \log \varepsilon(d)}{\sqrt{d}} \prod_{p \mid f}\left(1-\left(\frac{\Delta}{p}\right) p^{-1}\right)^{-1} .
\end{aligned}
$$

By (98) with $s=1$ and (101), we obtain after some simplification

$$
A(1, d)=\frac{h(d) \log \varepsilon(d)}{\sqrt{d}} \sum_{m \mid f} \frac{1}{m} \prod_{p \mid f / m}\left(1-p^{-1}\right)=\frac{h(d) \log \varepsilon(d)}{\sqrt{d}} .
$$

By (99) and (102), we obtain

$$
S_{1}=\frac{h(d) \log \varepsilon(d)}{2^{t(d)} \sqrt{d}} \cdot \frac{1}{s-1}+B(d)+O(s-1) .
$$

By (103), (97) and (92), we obtain the required result.

5. Evaluation of some definite integrals. Proofs of Theorems 5-10. Theorem 5, which is a consequence of Theorems 2 and 4, evaluates a class of definite integrals. Theorems 6-10 all follow from Theorem 5 .

Theorem 5. Let $G_{1}, G_{2} \in G(d)$. Then

$$
\int_{0}^{1}\left(\sum_{[a, b, c] \in G_{1}} E(a, b, c, t)-\sum_{[a, b, c] \in G_{2}} E(a, b, c, t)\right) d t
$$




$$
\begin{aligned}
= & \sum_{[a, b, c] \in G_{1}}\left(\frac{\pi^{2} \sqrt{d}}{6 a}-J(a, b, c)+\log \varepsilon(d) \log a\right) \\
& -\sum_{[a, b, c] \in G_{2}}\left(\frac{\pi^{2} \sqrt{d}}{6 a}-J(a, b, c)+\log \varepsilon(d) \log a\right) \\
& -\sqrt{d}\left(\beta\left(d, G_{1}\right)-\beta\left(d, G_{2}\right)\right),
\end{aligned}
$$

where all forms $(a, b, c)$ are chosen so that $a>0$,

$$
E(a, b, c, t)=\sum_{l=1}^{[(g-1) / 2]}\left(F(\alpha, l, t, g)+F\left(\alpha^{\prime}, l, t, g\right)\right)+\left(1+(-1)^{g}\right) \frac{\log \left(1+t^{\varepsilon}\right)}{1+t}
$$

and

$$
\begin{aligned}
& J(a, b, c) \\
& =-2 \sum_{l=1}^{[(g-1) / 2]}\left(\log (2 \sin (\pi l / g)) \log (2|\sin (\pi l \alpha / g)|)-\left(\frac{\pi}{2}-\frac{\pi l}{g}\right)\left(\frac{\pi}{2}-\frac{\pi l^{*}}{g}\right)\right) \\
& \quad-\frac{1+(-1)^{g}}{2} \log ^{2} 2 .
\end{aligned}
$$

Proof. In this proof, all forms $(a, b, c)$ satisfy $a>0$. We also write $\varepsilon=$ $\varepsilon(d)$. By Theorem 2, we have

$$
\begin{aligned}
\sum_{n=1}^{\infty} \frac{R_{G}(n, d)}{n^{s}=} & \sum_{[a, b, c] \in G} Z_{(a, b, c)}(s) \\
= & \sum_{[a, b, c] \in G}\left(\frac{\log \varepsilon}{\sqrt{d}} \cdot \frac{1}{s-1}+V(d)+\frac{\pi^{2}}{6 a}\right. \\
& \left.+\frac{\log \varepsilon \log a}{\sqrt{d}}-\frac{1}{\sqrt{d}} W_{(a, b, c)}\right)+O(s-1) \\
= & \frac{h(d) \log \varepsilon}{2^{t(d)} \sqrt{d}} \cdot \frac{1}{s-1}+\frac{V(d) h(d)}{2^{t(d)}} \\
& +\sum_{[a, b, c] \in G}\left(\frac{\pi^{2}}{6 a}+\frac{\log a \log \varepsilon}{\sqrt{d}}-\frac{1}{\sqrt{d}} W_{(a, b, c)}\right)+O(s-1) .
\end{aligned}
$$

By comparing with Theorem 4, we obtain

$$
B(d)+\beta(d, G)=\frac{V(d) h(d)}{2^{t(d)}}+\sum_{[a, b, c] \in G}\left(\frac{\pi^{2}}{6 a}+\frac{\log \varepsilon \log a}{\sqrt{d}}-\frac{1}{\sqrt{d}} W_{(a, b, c)}\right),
$$

which is (15). Thus 


$$
\begin{aligned}
\beta\left(d, G_{1}\right)-\beta\left(d, G_{2}\right)= & \sum_{[a, b, c] \in G_{1}}\left(\frac{\pi^{2}}{6 a}+\frac{\log \varepsilon \log a}{\sqrt{d}}-\frac{1}{\sqrt{d}} W_{(a, b, c)}\right) \\
& -\sum_{[a, b, c] \in G_{2}}\left(\frac{\pi^{2}}{6 a}+\frac{\log \varepsilon \log a}{\sqrt{d}}-\frac{1}{\sqrt{d}} W_{(a, b, c)}\right),
\end{aligned}
$$

which is (16). The result follows on noting that

$$
W_{(a, b, c)}=\int_{0}^{1} E(a, b, c, t) d t+J(a, b, c)
$$

and rearranging terms.

We now set

$$
D=d y_{0}(d)^{2}
$$

Then $\varepsilon(D)=\varepsilon(d)=\varepsilon$ and $y_{0}(D)=1$. Since $D+4$ is a square, we have $D+4 \equiv 0,1,4$ or $9(\bmod 16)$, so that $D \equiv 12,13,0$ or $5(\bmod 16)$. We are only interested in those $D$ for which $H(D)$ contains a class of the type $[2, b, c]$ or $[4, b, c]$. This rules out $D \equiv 5(\bmod 8)$, and so we are only interested in the cases $D \equiv 12(\bmod 16)$ and $D \equiv 0(\bmod 16)$. In the case $D \equiv 0(\bmod 16)$, we also have $\varepsilon(D / 4)=\varepsilon$ and $y_{0}(D / 4)=2$.

If $D$ is a positive integer such that $D \equiv 12(\bmod 16), D+4$ is a square and $H(D)$ has one class per genus, then we show in Theorem 6 that we can explicitly evaluate $\int_{0}^{1} \frac{\log \left(1+t^{\varepsilon}\right)}{1+t} d t$.

If $D$ is a positive integer such that $D \equiv 0(\bmod 16), D+4$ is a square, $D / 4 \equiv 8(\bmod 16)$ and $H(D)$ has one class per genus, then we show in Theorems 8 and 9 that we can evaluate explicitly both of the integrals $\int_{0}^{1} \frac{\log \left(1+t^{\varepsilon}\right)}{1+t} d t$ and $\int_{0}^{1} \frac{\tan ^{-1}\left(t^{\varepsilon}\right)}{1+t^{2}} d t$.

Before continuing we note the values of $E(a, b, c, t)$ and $J(a, b, c)$ for $g=1,2$ and 4 , which we shall need later.

If $g=1$, we have $E(a, b, c, t)=J(a, b, c)=0$.

If $g=2$, we have

$$
E(a, b, c, t)=2 \cdot \frac{\log \left(1+t^{\varepsilon}\right)}{1+t}, \quad J(a, b, c)=-\log ^{2} 2 .
$$

If $g=4$, we have

$$
\begin{aligned}
E(a, b, c, t) & =2 \cdot \frac{t \log \left(1+t^{2 \varepsilon}\right)-2(-1)^{(\alpha-1) / 2} \tan ^{-1}\left(t^{\varepsilon}\right)}{1+t^{2}}+2 \cdot \frac{\log \left(1+t^{\varepsilon}\right)}{1+t} \\
J(a, b, c) & =-\frac{3}{2} \log ^{2} 2+(-1)^{(\alpha-1) / 2} \frac{\pi^{2}}{8} .
\end{aligned}
$$

The next result is a slight modification of a result of Chowla ([2], [4: p. 967]). It is useful in proving that certain form classes are not equal. 
LEMMA 17. Let $k$ and $m$ be integers with $k>1, m$ not a square and $-(2 k-2)<m<2 k+2$. Then the equation

$$
x^{2}-\left(k^{2}-1\right) y^{2}=m
$$

has no solution in positive integers $x$ and $y$.

Proof. We suppose that (105) has a solution in positive integers $x$ and $y$. Let $\left(x_{1}, y_{1}\right)$ be the solution in positive integers to (105) for which $y_{1}$ is least. Let

$$
x_{2}=\left|k x_{1}-\left(k^{2}-1\right) y_{1}\right|, \quad y_{2}=\left|x_{1}-k y_{1}\right| .
$$

Then $x_{2}^{2}-\left(k^{2}-1\right) y_{2}^{2}=x_{1}^{2}-\left(k^{2}-1\right) y_{1}^{2}=m$. If $y_{2}=0$ then $m=x_{2}^{2}$, a contradiction. Thus, $y_{2} \geq 1$. If $x_{2}=0$, we have

$$
m=-\left(k^{2}-1\right) y_{2}^{2} \leq-\left(k^{2}-1\right) \leq-(2 k-2),
$$

a contradiction. Thus $x_{2}>0$. Hence, by the minimality of $y_{1}$, we have $y_{2} \geq y_{1}$. Thus, either $x_{1}-k y_{1} \geq y_{1}$ or $x_{1}-k y_{1} \leq-y_{1}$. If $x_{1}-k y_{1} \geq y_{1}$, we have

$$
m=x_{1}^{2}-\left(k^{2}-1\right) y_{1}^{2} \geq\left((k+1)^{2}-\left(k^{2}-1\right)\right) y_{1}^{2}=(2 k+2) y_{1}^{2} \geq 2 k+2,
$$

a contradiction. Similarly if $x_{1}-k y_{1} \leq-y_{1}$, we have $m \leq-(2 k-2)$, which is a contradiction.

First we consider the case $D \equiv 12(\bmod 16)$. For a positive integer $D$, it is easily checked that $D \equiv 12(\bmod 16)$ with $D+4$ a square if and only if $D=4\left(4 l^{2}-1\right)$ for some positive integer $l$.

Lemma 18. Let $D=4\left(4 l^{2}-1\right)$ for some positive integer $l$. Then

$$
\left[1,0,-\frac{D}{4}\right] \neq\left[2,2, \frac{4-D}{8}\right]
$$

in $H(D)$.

Proof. If $[1,0,-D / 4]=[2,2,(4-D) / 8]$, we have

$$
2=x^{2}-\frac{D}{4} y^{2}=x^{2}-\left(4 l^{2}-1\right) y^{2}
$$

for some positive integers $x, y$. But, by Lemma 17, equation (106) has no solution in positive integers since $2<2(2 l)+2$.

Theorem 6. Let $D=4\left(4 l^{2}-1\right)$ for some positive integer $l$ and suppose that $H(D)$ has one class per genus. Let $G_{1}$ be the genus containing $[1,0,-D / 4]$ and let $G_{2}$ be the genus containing $[2,2,(4-D) / 8]$. Then

$$
\int_{0}^{1} \frac{\log \left(1+t^{\varepsilon}\right)}{1+t} d t=\frac{\sqrt{D}}{2}\left(\beta\left(D, G_{1}\right)-\beta\left(D, G_{2}\right)\right)-\frac{\pi^{2} \sqrt{D}}{24}+\frac{\log 2 \log 2 \varepsilon}{2},
$$

where $\varepsilon=\varepsilon(D)=2 l+\sqrt{4 l^{2}-1}$. 
Proof. We observe that $G_{1} \neq G_{2}$ by Lemma 18. The result follows on using Theorem 5, noting that $y_{0}(D)=1, g=1$ for the form $(1,0,-D / 4)$, and $g=2$ for the form $(2,2,(4-D) / 8)$ and using the values of $E(a, b, c, t)$ and $J(a, b, c)$ given just before Lemma 17 .

The following are the first few cases where the conditions of Theorem 6 are satisfied so that we can calculate $\int_{0}^{1} \frac{\log \left(1+t^{\varepsilon}\right)}{1+t} d t$ :

- $l=1, D=12, \varepsilon=2+\sqrt{3}$,

- $l=2, D=60, \varepsilon=4+\sqrt{15}$,

- $l=3, D=140, \varepsilon=6+\sqrt{35}$,

- $l=4, D=252, \varepsilon=8+\sqrt{63}$,

- $l=6, D=572, \varepsilon=12+\sqrt{143}$,

- $l=7, D=780, \varepsilon=14+\sqrt{195}$.

Theorem 7.

$$
\begin{aligned}
\int_{0}^{1} \frac{\log \left(1+t^{2+\sqrt{3}}\right)}{1+t} d t= & \frac{\pi^{2}}{12}(1-\sqrt{3})+\log 2 \log (1+\sqrt{3}) . \\
\int_{0}^{1} \frac{\log \left(1+t^{4+\sqrt{15}}\right)}{1+t} d t= & \frac{\pi^{2}}{12}(2-\sqrt{15})+\log \left(\frac{1+\sqrt{5}}{2}\right) \log (2+\sqrt{3}) \\
& +\log 2 \log (\sqrt{3}+\sqrt{5}) . \\
\int_{0}^{1} \frac{\log \left(1+t^{6+\sqrt{35}}\right)}{1+t} d t= & \frac{\pi^{2}}{12}(3-\sqrt{35})+\log \left(\frac{1+\sqrt{5}}{2}\right) \log (8+3 \sqrt{7}) \\
& +\log 2 \log (\sqrt{5}+\sqrt{7}) . \\
\int_{0}^{1} \frac{\log \left(1+t^{8+\sqrt{63}}\right)}{1+t} d t= & \frac{\pi^{2}}{12}(4-\sqrt{63})+\log \left(\frac{5+\sqrt{21}}{2}\right) \log (2+\sqrt{3}) \\
& +\log 2 \log (3+\sqrt{7}) . \\
\int_{0}^{1} \frac{\log \left(1+t^{12+\sqrt{143})}\right.}{1+t} d t= & \frac{\pi^{2}}{12}(6-\sqrt{143})+\log \left(\frac{3+\sqrt{13}}{2}\right) \log (10+3 \sqrt{11}) \\
& +\log 2 \log (\sqrt{11}+\sqrt{13}) . \\
& \frac{\pi^{2}}{12}(7-\sqrt{195})+\log \left(\frac{1+\sqrt{5}}{2}\right) \log (25+4 \sqrt{39}) \\
& +\log \left(\frac{3+\sqrt{13}}{2}\right) \log (4+\sqrt{15}) \\
& +\log 2 \log (\sqrt{15}+\sqrt{13}) .
\end{aligned}
$$


The second, third and fifth integrals in Theorem 7 are due to Herglotz $[11$, p. 14].

We now turn to the case $D \equiv 0(\bmod 16)$. Let $D$ be a positive integer. Then $D \equiv 0(\bmod 16)$ with $D+4$ a square if and only if $D=16\left(l^{2}+l\right)$ for some positive integer $l$. If $D$ has this form, then $D \equiv 0(\bmod 32)$ and

$$
\frac{D}{4} \equiv \begin{cases}0(\bmod 16) & \text { if } l \equiv 0 \text { or } 3(\bmod 4), \\ 8(\bmod 16) & \text { if } l \equiv 1 \text { or } 2(\bmod 4) .\end{cases}
$$

Lemma 19. Let $D=16\left(l^{2}+l\right)$ for some positive integer $l$ with $l \equiv 1$ or $2(\bmod 4)$. Then $H(D)$ and $H(D / 4)$ have the same number of classes per genus.

Proof. Since $D+4$ is a square, we have $\varepsilon(D)=\varepsilon(D / 4)$. Hence (96) gives

$$
\frac{h(D)}{h(D / 4)}=2 \text {. }
$$

Since $D \equiv 0(\bmod 32)$, we have $t(D)=\omega(D)$. Since $D / 4 \equiv 8(\bmod 16)$, we have $t(D / 4)=\omega(D / 4)-1=\omega(D)-1$. Hence $t(D)=1+t(D / 4)$. Thus

$$
\frac{h(D / 4)}{2^{t(D / 4)}}=\frac{h(D) / 2}{2^{t(D / 4)}}=\frac{h(D)}{2^{t(D)}}
$$

as required.

Lemma 20. Let $D=16\left(l^{2}+l\right)$ for some positive integer $l$. Then

$$
[1,0,-D / 4] \neq[4,4,(16-D) / 16] \quad \text { in } H(D) \text {. }
$$

Proof. Suppose that $[1,0,-D / 4]=[4,4,(16-D) / 16]$. Then there exist coprime integers $\alpha, \gamma$ such that $\alpha^{2}-D \gamma^{2} / 4=4$. Thus

$$
\frac{\alpha+\gamma \sqrt{D / 4}}{2}= \pm \varepsilon(D / 4)^{n},
$$

for some integer $n$. But this gives

$$
\alpha+\gamma \sqrt{D / 4}= \pm 2(2 l+1+\sqrt{D / 4})^{n},
$$

so that $\alpha$ and $\gamma$ are even, a contradiction.

Lemma 21. Let $D=16\left(l^{2}+l\right)$ for some positive integer $l$ with $l \equiv 1$ or $2(\bmod 4)$. Then $[1,0,-D / 16] \neq[2,0,-D / 32]$ in $H(D / 4)$ except if $l=1$.

Proof. If $l=1$, we have $[1,0,-2]=[2,0,-1]$ in $H(8)$. If $l>1$ and $[1,0,-D / 16]=[2,0,-D / 32]$ in $H(D / 4)$, then there exist positive integers $x, y$ with $x^{2}-D y^{2} / 16=2$. Hence

$$
8=(2 x)^{2}-\frac{D}{4} y^{2}=u^{2}-\left((2 l+1)^{2}-1\right) v^{2}
$$


for some positive integers $u, v$. But, by Lemma 17, the equation (107) has no solution in positive integers since $8<2(2 l+1)+2$.

Theorem 8. Let $D=16\left(l^{2}+l\right)$ for some positive integer $l$. Suppose that $H(D)$ has one class per genus. Let $G_{1}$ be the genus containing $[1,0,-D / 4]$ and let $G_{2}$ be the genus containing $[4,4,(16-D) / 16]$. Then

$$
\begin{aligned}
& \int_{0}^{1} \frac{3 t \log \left(1+t^{2 \varepsilon}\right)+2(-1)^{l} \tan ^{-1}\left(t^{\varepsilon}\right)}{1+t^{2}} d t \\
& \quad=\frac{\sqrt{D}}{2}\left(\beta\left(D, G_{1}\right)-\beta\left(D, G_{2}\right)\right)-\frac{\pi^{2} \sqrt{D}}{16}+(-1)^{l} \frac{\pi^{2}}{16}+\log 2 \log \left(2^{3 / 4} \varepsilon\right),
\end{aligned}
$$

where $\varepsilon=\varepsilon(D)=2 l+1+\sqrt{D / 4}=2 l+1+\sqrt{4 l^{2}+4 l}$.

Proof. From Lemma 20 we see that $G_{1} \neq G_{2}$. We have $y_{0}(D)=1$. For the form $(1,0,-D / 4)$, we have $g=1$. For the form $(4,4,(16-D) / 16)$, we have $g=4, \alpha=2 l-1$. Using these facts together with the values of $E(a, b, c, t)$ and $J(a, b, c)$ given before Lemma 17 and the relation

$$
\int_{0}^{1} \frac{\log \left(1+t^{\varepsilon}\right)}{1+t} d t=2 \int_{0}^{1} \frac{t \log \left(1+t^{2 \varepsilon}\right)}{1+t^{2}} d t
$$

in Theorem 5 gives the required result.

In a similar manner, we obtain

Theorem 9. Let $D=16\left(l^{2}+l\right)$ for some positive integer $l$ with $l \equiv 1$ or $2(\bmod 4)($ so that $D / 4 \equiv 8(\bmod 16))$. Let $H(D)$ have one class per genus so that $H(D / 4)$ also has one class per genus by Lemma 19. In $H(D / 4)$, let $\widehat{G}_{1}$ be the genus containing $[1,0,-D / 16]$ and let $\widehat{G}_{2}$ be the genus containing $[2,0,-D / 32]$. Then

$$
\begin{aligned}
& \int_{0}^{1} \frac{t \log \left(1+t^{2 \varepsilon}\right)+2(-1)^{l+1} \tan ^{-1}\left(t^{\varepsilon}\right)}{1+t^{2}} d t \\
& =\frac{\sqrt{D}}{4}\left(\beta\left(D / 4, \widehat{G}_{1}\right)-\beta\left(D / 4, \widehat{G}_{2}\right)\right)-\frac{\pi^{2} \sqrt{D}}{48}+(-1)^{l+1} \frac{\pi^{2}}{16}+\frac{\log 2 \log (\sqrt{2} \varepsilon)}{2}, \\
& \text { where } \varepsilon=\varepsilon(D)=\varepsilon(D / 4)=2 l+1+\sqrt{4 l^{2}+4 l} .
\end{aligned}
$$

We note by Lemma 21 that $\widehat{G}_{1} \neq \widehat{G}_{2}$ if $l \neq 1$. If $D=16\left(l^{2}+l\right)$, for some positive integer $l$ with $l \equiv 1$ or $2(\bmod 4)$ and $H(D)$ has one class per genus, both Theorems 8 and 9 are applicable. Thus we can calculate both

$$
\int_{0}^{1} \frac{\log \left(1+t^{\varepsilon}\right)}{1+t} d t=2 \int_{0}^{1} \frac{t \log \left(1+t^{2 \varepsilon}\right)}{1+t^{2}} d t \quad \text { and } \int_{0}^{1} \frac{\tan ^{-1}\left(t^{\varepsilon}\right)}{1+t^{2}} d t .
$$


The following are the first few cases where this happens:

- $l=1, D=32, \varepsilon=3+\sqrt{8}$,

- $l=2, D=96, \varepsilon=5+\sqrt{24}$,

- $l=5, D=480, \varepsilon=11+\sqrt{120}$,

- $l=6, D=672, \varepsilon=13+\sqrt{168}$.

Applying Theorems 8 and 9 in these cases, we obtain

THEOREM 10.

$$
\begin{aligned}
& \int_{0}^{1} \frac{\log \left(1+t^{3+\sqrt{8}}\right)}{1+t} d t=\frac{\pi^{2}}{24}(3-\sqrt{32})+\frac{1}{2} \log 2 \log \left(2(3+\sqrt{8})^{3 / 2}\right), \\
& \int_{0}^{1} \frac{\tan ^{-1}\left(t^{3+\sqrt{8}}\right)}{1+t^{2}} d t=\frac{1}{16} \log 2 \log (3+\sqrt{8}) . \\
& \int_{0}^{1} \frac{\log \left(1+t^{5+\sqrt{24}}\right)}{1+t} d t=\frac{\pi^{2}}{24}(5-\sqrt{96})+\frac{1}{2} \log (1+\sqrt{2}) \log (2+\sqrt{3}) \\
& +\frac{1}{2} \log 2 \log \left(2(5+\sqrt{24})^{3 / 2}\right), \\
& \int_{0}^{1} \frac{\tan ^{-1}\left(t^{5+\sqrt{24}}\right)}{1+t^{2}} d t=\frac{1}{8} \log (1+\sqrt{2}) \log (2+\sqrt{3})-\frac{1}{16} \log 2 \log (5+\sqrt{24}) . \\
& \int_{0}^{1} \frac{\log \left(1+t^{11+\sqrt{120}}\right)}{1+t} d t=\frac{\pi^{2}}{24}(11-\sqrt{480})+\frac{1}{2} \log (1+\sqrt{2}) \log (4+\sqrt{15}) \\
& +\frac{1}{2} \log (2+\sqrt{3}) \log (3+\sqrt{10}) \\
& +\frac{1}{2} \log \left(\frac{1+\sqrt{5}}{2}\right) \log (5+\sqrt{24}) \\
& +\frac{1}{2} \log 2 \log \left(2(11+\sqrt{120})^{3 / 2}\right) \text {, } \\
& \int_{0}^{1} \frac{\tan ^{-1}\left(t^{11+\sqrt{120}}\right)}{1+t^{2}} d t=-\frac{1}{8} \log (1+\sqrt{2}) \log (4+\sqrt{15}) \\
& -\frac{1}{8} \log (2+\sqrt{3}) \log (3+\sqrt{10}) \\
& +\frac{3}{8} \log \left(\frac{1+\sqrt{5}}{2}\right) \log (5+\sqrt{24}) \\
& +\frac{1}{16} \log 2 \log (11+\sqrt{120}) \text {. }
\end{aligned}
$$




$$
\begin{aligned}
& \int_{0}^{1} \frac{\log \left(1+t^{13+\sqrt{168}}\right)}{1+t} d t=\frac{\pi^{2}}{24}(13-\sqrt{672}) \\
& +\frac{1}{2} \log (1+\sqrt{2}) \log \left(\frac{5+\sqrt{21}}{2}\right) \\
& +\frac{1}{4} \log (2+\sqrt{3}) \log (15+\sqrt{224}) \\
& +\frac{1}{4} \log (5+\sqrt{24}) \log (8+\sqrt{63}) \\
& +\frac{1}{2} \log 2 \log \left(2(13+\sqrt{168})^{3 / 2}\right), \\
& \int_{0}^{1} \frac{\tan ^{-1}\left(t^{13+\sqrt{168}}\right)}{1+t^{2}} d t=-\frac{3}{8} \log (1+\sqrt{2}) \log \left(\frac{5+\sqrt{21}}{2}\right) \\
& +\frac{1}{16} \log (2+\sqrt{3}) \log (15+\sqrt{224}) \\
& +\frac{1}{16} \log (5+\sqrt{24}) \log (8+\sqrt{63}) \\
& -\frac{1}{16} \log 2 \log (13+\sqrt{168}) \text {. }
\end{aligned}
$$

\section{References}

[1] D. A. Buell, Binary Quadratic Forms, Springer, New York, 1989.

[2] S. Chowla, On the inequality $\left|x^{2}-y^{2}-2 x y k\right| \geq 2 k(x, y, k$ odd $)$, Norske Vid. Selsk. Forh. (Trondheim) 34 (1961), 91. [Chowla's Collected Papers, Vol. II, p. 967.]

[3] - Remarks on class-invariants and related topics, in: 1963 Calcutta Math. Soc. Golden Jubilee Commemoration Vol. (1958/59), Part II, Calcutta Math. Soc., Calcutta, 361-372. [Chowla's Collected Papers, Vol. III, 1008-1019.]

[4] -, Collected Papers (3 Volumes), ed. by J. G. Huard and K. S. Williams, Centre de Recherches Math., Univ. Montréal, 1999.

[5] S. Chowla and A. Selberg, On Epstein's zeta function (I), Proc. Nat. Acad. Sci. U.S.A. 35 (1949), 371-374. [Chowla's Collected Papers, Vol. II, 719-722.]

[6] C. Deninger, On the analogue of the formula of Chowla and Selberg for real quadratic fields, J. Reine Angew. Math. 351 (1984), 171-191.

[7] P. G. L. Dirichlet, Vorlesungen über Zahlentheorie, Chelsea, New York, 1968.

[8] P. Epstein, Zur Theorie allgemeiner Zetafunctionen, Math. Ann. 56 (1903), 615644.

[9] D. R. Estes and G. Pall, Spinor genera of binary quadratic forms, J. Number Theory 5 (1973), 421-432.

[10] I. S. Gradshteyn and I. M. Ryzhik, Table of Integrals, Series, and Products, 5th ed., Academic Press, 1994.

[11] G. Herglotz, Über die Kroneckersche Grenzformel für reele, quadratische Körper. I., Ber. d. Sacks. Akad. d. Wiss. zu Leipzig 75 (1923), 3-14.

[12] L.-K. Hua, Introduction to Number Theory, Springer, Berlin, 1982. 
[13] J. G. Huard, P. Kaplan and K. S. Williams, The Chowla-Selberg formula for genera, Acta Arith. 73 (1995), 271-301.

[14] A. Selberg and S. Chowla, On Epstein's zeta-function, J. Reine Angew. Math. 227 (1967), 86-110. [Chowla's Collected Papers, Vol. III, 1101-1125.]

[15] C. L. Siegel, Advanced Analytic Number Theory, Tata Inst. Fund. Research, Bombay, 1980.

[16] D. Zagier, A Kronecker limit formula for real quadratic fields, Math. Ann. 213 (1975), 153-184.

Centre for Research in Algebra and Number Theory

School of Mathematics and Statistics

Carleton University

Ottawa, Ontario K1S 5B6, Canada

E-mail: hmuzaffa@math.carleton.ca

williams@math.carleton.ca 